Classification

Physics Abstracts

$68.55-68.60-73.60 \mathrm{D}$

\title{
Le matériau silicium sur saphir en France. Revue des propriétés physico-chimiques et électriques
}

\author{
S. Cristoloveanu, G. Ghibaudo et G. Kamarinos \\ Laboratoire de physique des composants à semiconducteurs, (ERA-CNRS 659), ENSERG, \\ 23, avenue des Martyrs, 38031 Grenoble Cedex, France
}

(Reçu le 7 mars 1983, révisé le 18 octobre, accepté le 21 octobre 1983)

\begin{abstract}
Résumé. - Nous présentons une synthèse des travaux effectués en France sur la caractérisation des couches minces de silicium épitaxié sur des substrats de corindon (" saphir »). Une revue exhaustive des méthodes et des résultats concernant les propriétés physico-chimiques (défauts, impuretés, contamination, ...) et électriques (processus de transport, de recombinaison, de piégeage, ...) du SOS est présentée. Il apparaît que sur le plan physique les éléments clefs sont : (i) l'existence d'une région de transition d'épaisseur 100 à $150 \AA$, située à l'interface $\mathrm{Si}_{-} \mathrm{Al}_{2} \mathrm{O}_{3}$, responsable pour l'essentiel des courants de fuite des transistors MOS/SOS, (ii) la non-uniformité des couches et leur caractère inhomogène lié à la présence d'un profil de défauts croissant vers l'interface $\mathrm{Si}_{-} \mathrm{Al}_{2} \mathrm{O}_{3}$ et qui s'accompagne d'une diminution notable de la mobilité et de la durée de vie des porteurs, (iii) les fortes contraintes d'interface qui provoquent une modification appréciable de la configuration énergétique et des paramètres de transport par rapport au Si massif, (iv) l'autodopage du film et (v) les fluctuations de potentiel. La cohérence des résultats émanant d'études indépendantes sur le matériau SOS non processé ou sur des dispositifs SOS est ensuite soulignée. Compte tenu de la pluralité des techniques et des conditions de mesure employées nous discutons également leurs pertinence, précision et limitations, ainsi que leur intérêt pour la caractérisation des couches minces issues d'autres technologies silicium sur isolant.
\end{abstract}

\begin{abstract}
We present an overview of investigations conducted in France for the assessment of thin Si films epitaxied on sapphire substrates. Techniques and relevant data concerning physico-chemical properties (defects, impurities, contamination, ...) and electrical features (transport, recombination, trapping, ...) in SOS are reviewed extensively. The key physical parameters are : (i) existence of a transition layer 100-150 $\AA$ thick, situated at the $\mathrm{Si}-\mathrm{Al}_{2} \mathrm{O}_{3}$ interface, which is responsible for important leakage currents in MOS/SOS transistors, (ii) film nonuniformity connected with an increasing profile of defects towards the $\mathrm{Si}_{-} \mathrm{Al}_{2} \mathrm{O}_{3}$ interface and resulting in a noticeable degradation of carrier mobility and lifetime, (iii) lateral stress at the film-substrate interface, the effect of which is to modify both the band energy configuration and the transport parameters as compared to bulk $\mathrm{Si}$, (iv) autodoping and (v) potentiel fluctuations. The consistency of different results derived from independent measurements performed on non-processed SOS films and on devices is emphasized. Finally, taking into account the great number of techniques and experimental conditions we also discuss their pertinence, precision or limitations as well as their interest for the characterization of other silicon on insulator technologies.
\end{abstract}

\section{Introduction.}

Les technologies Silicium Sur Isolant (SSI) ont été depuis longtemps considérées comme très intéressantes à bien des égards par rapport à la technologie des circuits intégrés au silicium massif monocristallin [1-4]. A l'heure actuelle le SOS (Silicon On Sapphire) se présente encore comme la plus avantageuse filière de SSI [5] car elle permet : i) une densité plus élevée d'intégration des circuits, ii) une résistance plus grande aux rayonnements ionisants et iii) une réduction des capacités parasites du substrat.

Ce sont les raisons pour lesquelles un effort considé- rable de développement de la filière SOS a été entrepris très tôt. A Grenoble, l'étude du SOS, obtenu par pyrolyse du silane $\left(\mathrm{SiH}_{4}\right)$ a commencé vers l'année 1964 aux laboratoires du CNRS $\left({ }^{1}\right)$ [6]. Cependant, ce n'est qu'après 1970 que ces études ont été intensifiées au LETI $\left({ }^{1}\right)$ où les premiers rapports de recherches sur le SOS apparaissent en 1973 [7].

( $\left.{ }^{1}\right)$ CNRS, Centre National de la Recherche Scientifique. LETI, Laboratoire d'Electronique et de Technologie de l'Informatique. 
L'analyse des propriétés des dépôts de silicium repose sur deux types d'investigation : caractérisation physico-chimique (microscopique) et caractérisation électrique. La caractérisation physico-chimique du matériau a permis de mettre au point le processus d'épitaxie. A ce stade de maturité, les limitations essentielles de la technologie SOS trouvaient leurs origines dans les imperfections électriques des couches (courant de fuite des transistors MOS, faible gain des transistors bipolaires latéraux, etc...). Dès 1978 une action concertée (GCIS $\left({ }^{2}\right)$ ) s'est fixé pour objectif d'une part, de compléter les études physico-chimiques et d'autre part, d'entreprendre la caractérisation électrique des couches. Les principaux traits de cette action sont :

- l'analyse fine de la surface des substrats $\mathrm{Al}_{2} \mathrm{O}_{3}$ à l'aide de la réflexion rasante des rayons $\mathrm{X}$.

- l'étude de la nucléation du SOS par microscopie électronique à balayage.

- l'étude de l'interface $\mathrm{Si} / \mathrm{Al}_{2} \mathrm{O}_{3}$.

- la caractérisation électrique des couches de SOS.

La synthèse des résultats obtenus en France sur le SOS et leur confrontation dans un contexte international $[8,9]$ montrent que le SOS français est au moins équivalent au SOS américain ou japonais et que les méthodes de caractérisation mises au point sont non seulement pertinentes pour le SOS mais peuvent être avantageusement utilisées pour d'autres filières SSI.

Cet article est divisé en trois principaux chapitres. Le premier est consacré à la présentation de l'étude structurelle du dépôt de silicium à différents stades de l'épitaxie par analyse physico-chimique. Le deuxième chapitre présente les résultats de la caractérisation électrique réalisée tant sur des couches de SOS que sur des dispositifs conventionnels ou spécifiques. Enfin le dernier chapitre conclut sur la cohérence des résultats et dégage des enseignements recueillis les méthodes optimales en vue de la caractérisation d'autres filières Silicium Sur Isolant.

\section{Préparation du silicium sur saphir et propriétés cristallines.}

2.1 Le SUBSTRAT ISOlant. - Le corindon a été choisi comme substrat du SOS essentiellement [10-13] en raison de sa configuration cristalline qui permet d'aboutir à une relative concordance des plans [11102] du substrat et [001] du silicium épitaxié. L'épitaxie du silicium n'est cependant pas "parfaite " car il existe un désaccord de maille résiduel de 4 à $12 \%$ selon la direction, qui contribue à la compression du film, partant aux propriétés électriques du matériau (cf. § 3).

La lame de corindon est minutieusement préparée (polissages mécaniques et décapages chimiques) avant l'épitaxie [12]; cette étape s'avère essentielle pour la qualité des couches de silicium obtenues. L'état de la

( ${ }^{2}$ GCIS, Groupement Circuits Intégrés au Silicium. surface du substrat est étudié à l'aide d'un diffractomètre de rayons $\mathrm{X}$ à double cristal ou à l'aide de la Microscopie Electronique à Balayage (MEB) [11]. $\mathrm{La}$ réflexion rasante des rayons $\mathrm{X}$ a mis en évidence d'une part une qualité exceptionnelle de la surface (rugosité ne dépassant pas $5 \AA \AA$, c'est-à-dire une monocouche) et d'autre part l'existence d'une couche d'interface, d'une vingtaine d'Angströms, de densité électronique différente de celle du corindon massif; sa composition moyenne semble proche de celle de l'oxyhydroxyde. Un traitement de $30 \mathrm{~min}$. dans l'hydrogène ne paraît pas modifier les caractéristiques géométriques de la couche superficielle mais entraîne une hydratation variable avec la profondeur sur $100 \AA$ environ [15]. Par ailleurs des études en spectroscopie Auger [16] ont montré que la contamination du substrat par les atomes de carbone est à l'origine d'une mauvaise nucléation.

2.2 Le DÉPôT DE SILICIUM. - Les dépôts de silicium sur corindon ont été réalisés au LETI au moyen d'un réacteur d'épitaxie [17]. Le silicium est déposé à partir de la phase vapeur à $980^{\circ} \mathrm{C}$ par pyrolyse du silane $\left(\mathrm{SiH}_{4}\right)$ en atmosphère d'hydrogène selon la réaction :

$$
\mathrm{SiH}_{4(\mathrm{gaz})} \rightarrow \mathrm{Si}_{\text {(sol) }}+2 \mathrm{H}_{2 \text { (gaz) }} \text {. }
$$

La vitesse de dépôt est de $2 \mu \mathrm{m} / \mathrm{min}$. [13, 16] et l'épaisseur standard utilisée dans la technologie des circuits intégrés sur SOS est actuellement de 0,65 $\mu \mathrm{m}$. Néanmoins pour les études menées, des dépôts jusqu'à $7 \mu \mathrm{m}$ d'épaisseur (mesurée par pyrométrie IR [10]), ont été réalisés. Une autre technique de dépôt du silicium par pulvérisation ionique a été proposée par le groupe de Vapaille [18]. Cette méthode, permettant entre autres une température de dépôt plus basse, n'a pas encore été exploitée pour la fabrication de composants intégrés sur SSI.

\subsection{CARACTÉRISATION PHYSICO-CHIMIQUE DU DÉPÔT} DE SILICIUM. - La caractérisation physico-chimique du matériau vise l'appréciation de la structure cristalline, du taux de défauts, du degré de contamination et de la cinétique de nucléation du matériau sur isolant.

L'observation des dépôts de Si non complètement coalescés par Microscopie Electronique en Transmission (MET) a efficacement contribué à l'étude des premières étapes de la nucléation montrant en particulier que [19] :

- l'existence d'îlots de silicium épitaxié dans deux orientations différentes, l'une minoritaire (quelques \%) (110), l'autre majoritaire (100). Au cours de la croissance du film, la fraction volumique des domaines (100) augmente régulièrement (Fig. 2.1);

- une forte distorsion du réseau de silicium (100) conduisant pendant les premiers stades du dépôt (épaisseur de l'ordre de $100 \AA$ ) à une certaine cohérence du réseau du silicium sur le plan de corindon. Des contraintes de l'ordre de $10^{12} \mathrm{dyn} / \mathrm{cm}^{2}$ ont été mesurées ; 


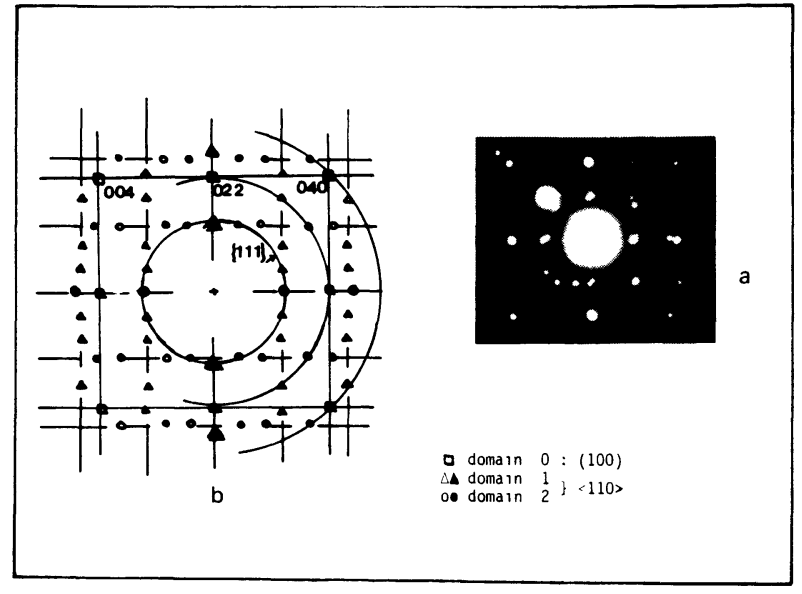

Fig. 2.1. - Figure de diffraction obtenue sur SOS $(a)$ et interprétation $(b)$ (d'après Hamar-Thibault [19]).

[Diffraction pattern obtained on SOS $(a)$ and interpretation (b) (after Hamar-Thibault [19]).]

- une densité très importante de mâcles (de l'ordre de $10^{9} \mathrm{~cm}^{-2}$ ) dans les divers îlots (100) et (110).

Pour les films "formés " c'est-à-dire d'épaisseur supérieure à $500 \AA$, les contraintes sont moins importantes (environ $10^{9} \mathrm{dyn} / \mathrm{cm}^{2}$ ) comme l'ont montré des

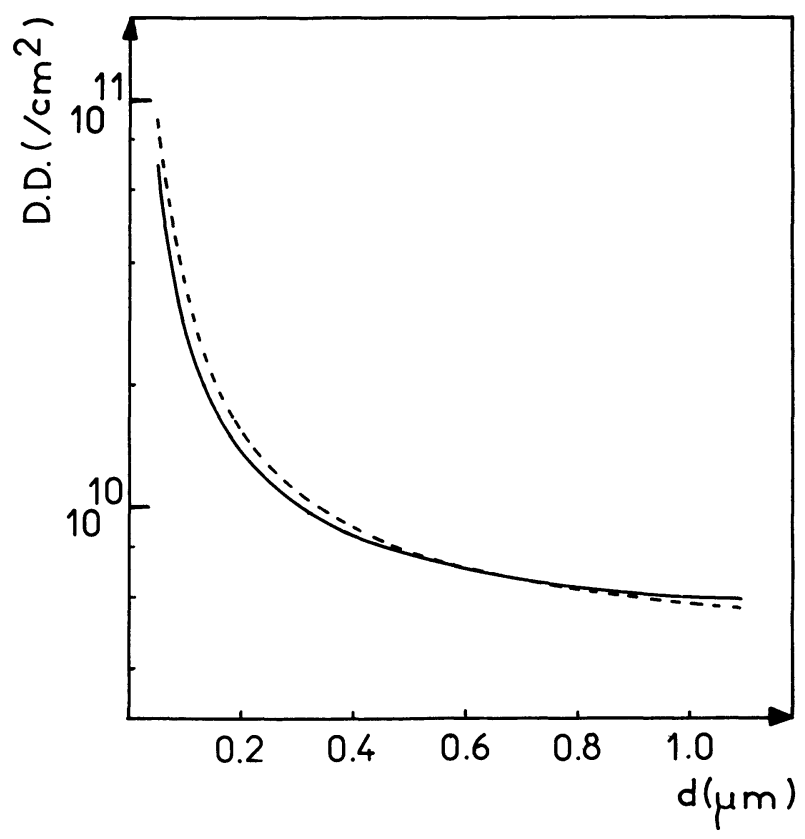

Fig. 2.2. - Profils de la densité de dislocations en fonction de la distance à l'interface silicium-corindon : (---) obtenue par interprétation simple de la mosaique en terme de dislocations, $(-)$ obtenue par la théorie de Wilkens (d'après Gonchond [13]).

[Dislocation density profiles as a function of distance from the Si-sapphire interface : (---) obtained by a simple interpretation of the mosaic structure in term of dislocation, (-) obtained using the Wilkens's theory (after Gonchond [13]).] expériences en spectroscopie Raman [20]. Les autres techniques le plus souvent utilisées sont :

- la microscopie électronique (MET et MEB) $[13,16,21,22]$,

- la diffraction des rayons $\mathrm{X}$ et plus particulièrement la technique de "Rocking curve " $[10,13]$,

- la diffraction électronique Rheed $[10,16]$,

- l'analyse par sonde ionique $[10,13,16,23]$,

- l'analyse par spectroscopie Auger [16].

Les études menées à l'aide des premières méthodes mentionnées ci-dessus indiquent que la couche de silicium est constituée d'un monocristal imparfait " mosaique ». La taille limite inférieure d'un bloc de mosaïque a été estimée par "Rocking curve" à $6000 \AA$ (dimension latérale) pour un film d'épaisseur de $0,64 \mu \mathrm{m}$ [13]. Les mêmes études ont montré que la taille inférieure du bloc mosaiqque diminue avec l'épaisseur du film.

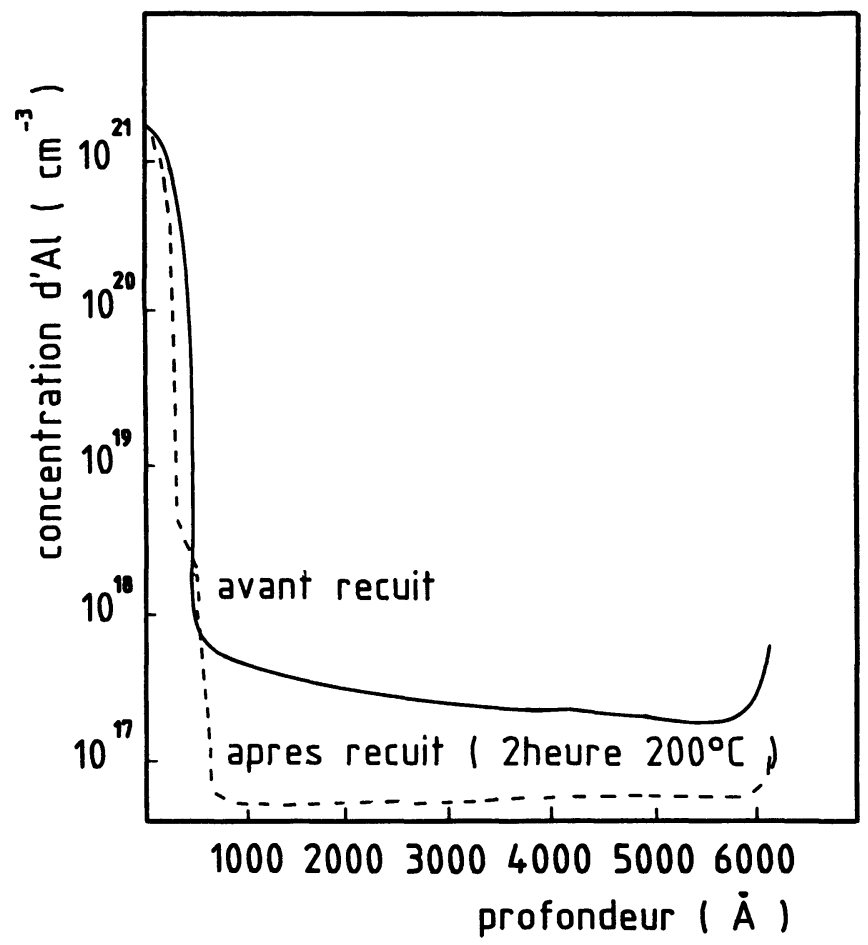

Fig. 2.3. - Profils d'aluminium dans le silicium sur corindon (d'après Trilhe et al. [24]).

[Aluminium profiles in SOS films (after Trilhe et al. [24]).]

Par ailleurs, Gonchond [13] a observé que la densité des dislocations et des défauts linéaires diminue spectaculairement dès qu'on s'éloigne de l'interface $\mathrm{Si} / \mathrm{Al}_{2} \mathrm{O}_{3}$ (Fig. 2.2). Les analyses menées à l'aide de la sonde ionique (SIMS) et par spectroscopie Auger $[13,16,22]$ ont clairement mis en évidence la contamination du silicium épitaxié par les atomes d'aluminium (provenant du corindon)(Fig. 2.3) ainsi que par le carbone [16]. 
3. Propriétés électriques des couches de silicium sur saphir.

\section{1 INFLUENCE DES CONTRAINTES SUR LES COEFFICIENTS} DE TRANSPORT.

3.1.1 Considérations générales. - Il est bien connu que les propriétés de transport dans le SOS diffèrent sensiblement de celles du silicium massif $[4,25,26]$. Ceci se manifeste par une dégradation des principaux paramètres de transport, qui résulte du caractère hétéroépitaxié du matériau, et qui se traduit par une diminution de la mobilité effective dans les couches $[26,27,28]$ et par une anisotropie notable $[29,30,31]$. Les raisons en sont d'une part, la qualité cristalline médiocre des films et d'autre part, la présence de fortes contraintes d'interface film-substrat. Ces contraintes, qui prennent naissance lors de la phase de croissance du film, proviennent pour l'essentiel de la différence entre les coefficients de dilatation thermique du Si et du saphir. Schlotterer [26] a, le premier, mis en évidence leur conséquence sur la mobilité des porteurs, en constatant une diminution (resp. augmentation) de la mobilité des couches de type $\mathbf{N}$ (resp. de type P) par rapport au Si massif. Hynecek [28], grâce à des études d'élastorésistance sur du SOS de type $\mathrm{N}$, a montré que la mobilité diminue de 20 à $40 \%$ sous l'effet des contraintes en analysant l'anisotropie de la mobilité et de la magnétorésistance (MR).

L'interprétation des résultats expérimentaux illustrant le rôle des contraintes sur les propriétés de transport, repose sur la modification de la structure des bandes d'énergie sous l'effet d'une compression quasi isotrope du film [28, 29,31]. En effet, on considère à juste titre que les contraintes film-substrat sont homogènes selon la direction d'épitaxie à cause de la faible épaisseur du film de Si devant celle du corindon (1 pour 300 ), et ne présentent pas plus de $9 \%$ d'anisotropie pour l'orientation considérée $\left((001) \mathrm{Si} /(1 \overline{1} 02) \mathrm{Al}_{2} \mathrm{O}_{3}\right)$ $[27,29]$. Il en résulte un écartement énergétique pour deux des 6 vallées de la bande de conduction, ainsi que la levée de la dégénérescence des trous lourds et des trous légers de la bande de valence. Récemment Englert et al. [20] ont déduit, grâce à des mesures de contrainte par spectroscopie Raman, que la séparation énergétique des vallées de la bande de conduction est de l'ordre de $75 \mathrm{meV}$. Cela explique la quasidépopulation des deux vallées de l'axe $\mathrm{o} z$, direction d'épitaxie des couches (Fig. 3.1).

3.1.2. Conséquences sur les coefficients de transport. Le point de départ dans l'étude des phénomènes de transport est constitué par l'équation de Boltzmann. La résolution donnée par Shibuya [33,34] permet d'obtenir le tenseur de conductivité électrique pour une induction magnétique $B$ d'intensité arbitraire (ne conduisant pas à des effets quantiques) et sous des hypothèses usuelles : anisotropie de masse effective, approximation du temps de relaxation, surface isoénergétique ellipsoïdale, faible champ électrique, cou- rant de diffusion négligeable et pas de contraintes extérieures. Par ailleurs, Herring et Vogt [35] ont montré que l'anisotropie du temps de relaxation peut être prise en compte comme une pondération de l'anisotropie de masse effective. Le tenseur de conductivité général $\sigma$ se présente comme la superposition des tenseurs $\sigma(i)$ qui correspondent à chacune des vallées énergétiques $(i)$ participant à la conduction. Ainsi dans le cas du Si massif (monocristallin) de type n, on somme les contributions équivalentes des 6 ellipsoïdes de la figure 3.1.

Dans les couches minces, le traitement rigoureux du problème est encore plus complexe car la connaissance des paramètres gouvernant les contraintes (coefficients anisotropes de dilatation thermique et constantes de réseau du film et du substrat) devient nécessaire. Tout en négligeant le désaccord des réseaux, Hughes [29] a déterminé le tenseur de piézorésistance du SOS, ce qui lui a permis d'analyser l'influence de la contraction thermique sur les valeurs de la mobilité des électrons et des trous, pour différentes orientations du Si et du Saphir.

Toutefois, lorsqu'on s'intéresse à la configuration usuelle du SOS, à savoir $(001) \mathrm{n}-\mathrm{Si} /(1 \overline{102}) \mathrm{Al}_{2} \mathrm{O}_{3}$, le calcul des différents coefficients magnétoélectriques peut être développé d'une manière simple, similaire à celle utilisée pour le Si massif. En se référant au schéma de la figure 3.1, Lee et al. [36] déterminent le tenseur général de conductivité $\sigma$ par addition des contributions quasi équivalentes des vallées (100), (100), (010), et $(0 \overline{1} 0)$ (parmi lesquelles se répartissent les électrons de la bande de conduction).

$$
\begin{aligned}
\sigma_{\alpha \beta} & =n e \delta_{\alpha \beta}\left(\overline{\left.\frac{u}{2 K} \sum_{i=x, y} \frac{K+(1-K) \delta_{\alpha i}}{D_{i}}\right)}-\right. \\
& -n e \sum_{\gamma=x, y, z} B_{\gamma} \delta_{\alpha \beta \gamma}\left(\overline{\left.\frac{u^{2}}{2 K} \sum_{i=x, y} \frac{1+(K-1) \delta_{\gamma i}}{D_{i}}\right)}+\right. \\
& +n e B_{\alpha} B_{\beta}\left(\overline{\left.\frac{u^{2}}{2 K} \sum_{i=x, y} \frac{1}{D_{i}}\right)}\right.
\end{aligned}
$$

Dans cette expression :

$n$ et $(-e)$ sont la concentration et la charge des électrons.

$K=m_{1} \tau_{\mathrm{t}} / m_{\mathrm{t}} \tau_{1}$ est le coefficient d'anisotropie dépendant des composantes longitudinales et transversales de la masse effective $\left(m_{1}, m_{\mathrm{t}}=m\right)$ et du temps de relaxation $\left(\tau_{1}, \tau_{\mathbf{t}}=\tau\right)$,

$u=e \tau / m$.

$\bar{A}$ dénote la moyenne énergétique usuelle de $A(\varepsilon)$,

$\delta_{\alpha \beta}$ est le symbole de Kronecker,

$\delta_{\alpha \beta \gamma}$ est égal à +1 pour des permutations directes de $(\alpha, \beta, \gamma)$ parmi les directions cristallographiques principales $(x, y, z)$, à -1 pour des permutations inverses et à 0 pour les autres cas.

A l'aide de la relation $J=\sigma E$ on peut expliciter aisément tout effet galvanomagnétique quelle que soit l'orientation des champs électrique $E$ et magnétique $B$ 


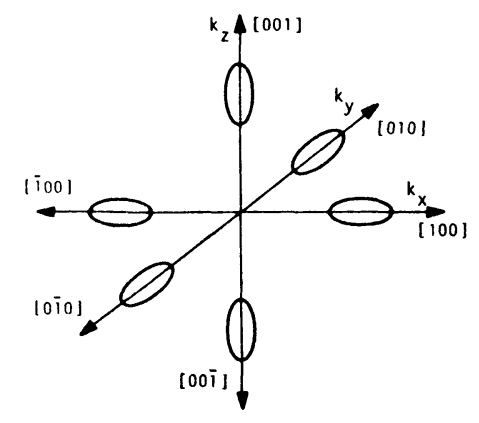

A

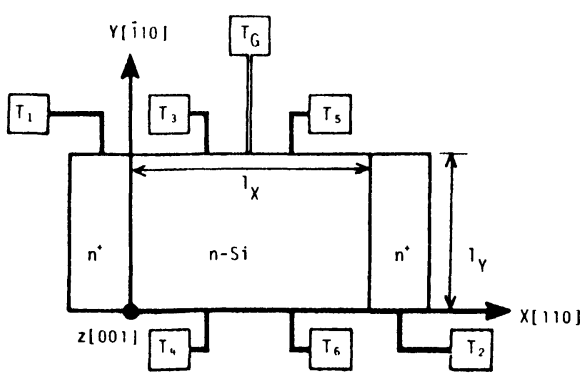

B

Fig. 3.1. - a) Bande de conduction du SOS sous l'effet des contraintes film-substrat (d'après LEE [32], Englert et al. [20]). b) Structure et orientation cristallographique des échantillons MOS-HALL.

[a) Stress induced configuration of SOS conduction band (after LEE [32], Englert et al. [20]). b) Structure and cristallographic orientation of MOS-HALL samples.]

extérieurs. Les exemples qui suivent sont relatifs à l'échantillon de la figure 3.1 et ont pour but d'illustrer les conséquences pratiques des contraintes; pour cela les résultats correspondant au SOS (considéré ici comme homogène $[36,37])$ seront comparés à ceux du Si massif [38].

Le tenseur de conductivité à $B=0$ reste diagonal mais ne se réduit plus à une constante, comme dans le cas du Si massif; ceci se traduit par l'apparition d'une anisotropie de la mobilité de dérive $\mu_{0}$ :

$\mu_{0}=-\bar{u}$ dans la direction épitaxiale $z$, et

$\mu_{0}=-\frac{\overline{K+1} u}{2 K}$ dans le plan $(x, y)$ du film

alors que dans le Si massif

$$
\mu_{0}=-\frac{\overline{(2 K+1) u}}{3 K} .
$$

Si on suppose que les contraintes ne modifient pas sensiblement les valeurs du coefficient d'anisotropie $(K \# 5$ à 7$)$, de la masse effective et des paramètres des collisions électroniques, il résulte que le rapport des mobilités dans le plan du film et dans le Si massif est égal à $3(K+1) / 2(2 K+1)$, ce qui équivaut à une dégradation de mobilité d'environ 20 à $25 \%[28,36]$.

La mobilité de Hall dans le SOS (à faible champ magnétique $B_{z}^{(001)} \perp J_{x}^{(110)}$ et pour $\left.K(\varepsilon)=\mathrm{Cte}\right)$ :

$$
\left(\mu_{\mathrm{H}}\right)_{110}^{001}=-\frac{2}{K+1} \frac{\bar{u}^{2}}{\bar{u}}
$$

est environ deux fois plus faible que dans le cas du $\mathrm{Si}$ massif où :

$$
\left(\mu_{\mathrm{H}}\right)_{110}^{001}=-\frac{K+2}{2 K+1} \frac{\bar{u}^{2}}{\bar{u}} .
$$

Par conséquent la réduction de la mobilité de Hall s'avère plus importante que celle affectant la mobilité de dérive; ainsi pour des collisions acoustiques le rapport $\mu_{\mathrm{H}} / \mu_{0}$ dans le SOS est voisin de $60 \%$ alors qu'il valait approximativement 1 dans le $\mathrm{Si}$. Ce résultat montre que la confusion usuelle entre les mobilités de dérive et de Hall constitue une importante source d'erreurs dans le SOS ; en effet, on s'est souvent fondé sur la faible mobilité de Hall pour sous-estimer la qualité cristalline du film.

L'effet de MR longitudinale dans la direction (110) est plus accentué dans le SOS. Ainsi le coefficient de magnétorésistance $\left(\rho_{\mathrm{B}} / \rho_{0}\right)_{110}^{110}$ tend vers une limite $[36,37]$ :

$$
\left[\frac{\rho_{\mathrm{B}}}{\rho_{0}}\right]_{110}^{110} \underset{B \rightarrow \infty}{\longrightarrow} \frac{(K+1)^{2}}{4 K}
$$

indépendante des processus collisionnels et plus forte d'environ $50 \%$ que dans le Si massif. Cette valeur de saturation est d'autant plus élevée que la mobilité est faible (car $\rho_{\mathrm{B}} / \rho_{0} \# \mu_{0} / \mu_{\mathrm{H}}$, cf. Eqs. 3.2-3.4) et pourrait augmenter indéfiniment avec $K$, alors qu'elle était limitée pour le Si massif à $200 \%$. Ceci suggère par ailleurs l'importance des mesures de MR longitudinale dans le SOS.

Quant à la MR transversale elle se sature dans le SOS à une valeur gouvernée uniquement par les processus de collisions et donc indépendante du coefficient d'anisotropie.

Ces résultats donnent une image succincte du rôle essentiel des contraintes dans le SOS. Nous verrons par la suite que l'interprétation cohérente de la réalité expérimentale exige de prendre également en compte l'inhomogénéité du film SOS.

3.2 PROPRIÉTÉS DE TRANSPORT GLOBALES DES FILMS. L'étude des propriétés de transport globales des films SOS repose sur la mesure des coefficients de transport tels que conductivité, photoconductivité, coefficient de Hall, magnétorésistance et pouvoir thermoélectrique dans une large gamme de température $(4 \mathrm{~K}<T<$ 
$380 \mathrm{~K})$ et de champ magnétique $(0<B<14 \mathrm{~T})$. Les échantillons SOS étudiés, de type $\mathbf{N}$ (dopés au phosphore) et de type $\mathbf{P}$ (dopés au bore), ont des dopages compris entre quelque $10^{15} \mathrm{~cm}^{-3}$ et quelque $10^{18} \mathrm{~cm}^{-3}$ pour des épaisseurs de Si épitaxié de 0,2 à $7 \mu \mathrm{m}$. Les résultats obtenus par ces investigations sont analysés par des modèles théoriques $[39,40]$ prenant en compte le caractère inhomogène des couches épitaxiées, à la fois dans la direction d'épitaxie et dans le plan du substrat. Les enseignements qui en découlent concernent les processus fondamentaux qui déterminent le transport électronique dans le matériau, tels que mécanismes de collisions des porteurs, fluctuations de potentiel à moyenne portée, nature dégénérée de la couche de transition à l'interface $\mathrm{Si}-\mathrm{Al}_{2} \mathrm{O}_{3}$.

3.2.1 Conductivité électrique. - La conductivitéélectrique des couches épitaxiées de $\mathrm{Si}$ a fait l'objet de nombreuses études. Roux et Bielle-Daspet [41] ont étudié la conductivité en fonction de la température sur des échantillons de SOS de type $\mathrm{P}$ pour différentes épaisseurs des couches (Fig. 3.2a). Ces résultats montrent d'une façon générale que, dans les couches plus minces, le maximum de la conductivité a tendance à se déplacer vers les hautes températures. Cela signifie qualitativement que les films ont une meilleure qualité électronique quand l'épaisseur de $\mathrm{Si}$ épitaxié augmente. Nous verrons par la suite que le comportement de la mobilité de Hall confirme cette affirmation ( $\$ 3.2$ ).

Ghibaudo et Kamarinos [40] ont étudié les variations de la conductivité avec la température sur du SOS de type $\mathrm{N}$ (Fig. 3.2b). Leur analyse basée sur un modèle bicouche [40, 43-45] prenant en compte l'inhomogénéité transversale naturelle du film, autorise à penser que, entre 77 et $360 \mathrm{~K}$, la conductivité est principalement gouvernée par les effets de mobilité dans la partie supérieure du film. Ils montrent qu'en combinant les processus de collisions sur les phonons acoustiques et sur les impuretés ionisées, les variations de la conductivité avec la température sont assez bien décrites. Ils notent cependant que bon nombre d'autres mécanismes de diffusion ont été suggérés $[4,25,46]$ et qu'à ce sujet l'unanimité est loin d'être atteinte.

Gris et Bello [42] ont mesuré la résistance " carré " (résistivité/épaisseur) de films SOS d'épaisseur variable à dopage (type $\mathrm{N}$ et $\mathrm{P}$ ) volumique sensiblement constant $\left(\# 5 \times 10^{19} \mathrm{~cm}^{-3}\right)$. En raison du fort dopage qui "masque " la contribution des défauts intrinsèques au processus de collision dans la couche épitaxiée, la dépendance de la résistance "carré " est quasi linéaire en fonction de l'inverse de l'épaisseur du film.

Enfin Robert et al. [39] ont effectué des mesures de conductivité électrique en fonction de la température, en obscurité $\sigma_{0}$ et sous lumière $\Delta \sigma$, sur du SOS de type N. Les résultats obtenus (Fig. 3.3) indiquent que le comportement de la conductivité correspond à un matériau dégénéré entre 4 et $40 \mathrm{~K}$. Pour des températures plus élevées, la conductivité en obscurité présente une énergie d'activation de l'ordre de $12 \mathrm{meV}$.

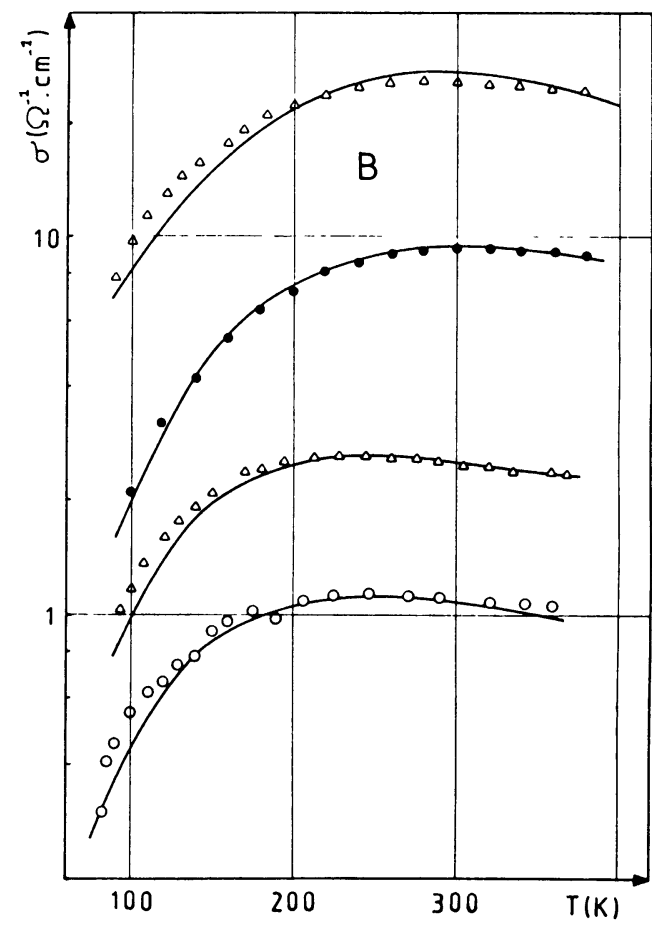

Fig. 3.2. - Variation de la conductivité en fonction de la température : a) SOS de type $\mathrm{P}$ (d'après Roux et Bielle-Daspet [41]), b) SOS de type $\mathrm{N}$ dopé de $10^{16}$ à $3 \times 10^{17} \mathrm{~cm}^{-3}$ (d'après Ghibaudo et Kamarinos [40]).

[Conductivity as a function of temperature : $a$ ) $\mathrm{P}$ type SOS (after Roux and Bielle-Daspet [41]), $b$ ) N type SOS with doping between $10^{16}$ and $3 \times 10^{17} \mathrm{~cm}^{-3}$ (after Ghibaudo and Kamarinos [40]).] 


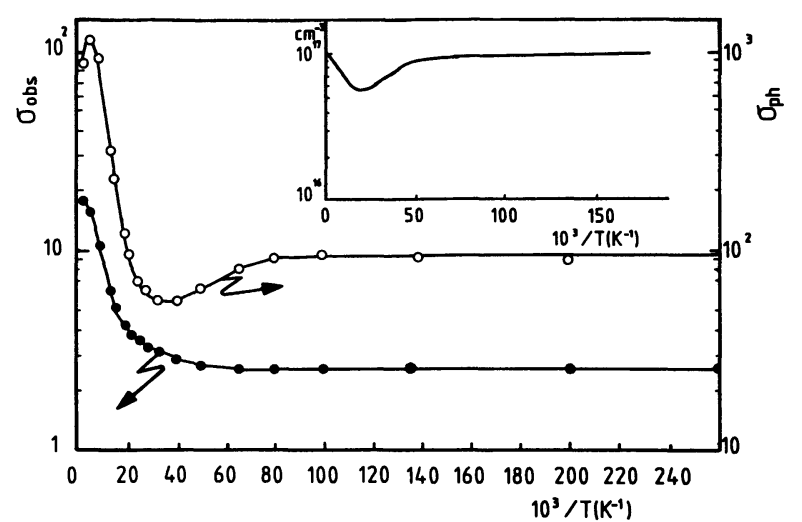

Fig. 3.3. - Variations de la conductivité en obscurité et/ou sous illumination avec la température pour du N-SOS. En encart : comportement théorique de la photoconductivité (d'après Robert et al. [39]).

[Dark conductivity and photoconductivity as a function of temperature for N-SOS. In insert : theoretical behaviour of photoconductivity (after Robert et al. [39]).]

La conductivité sous lumière, qui a un comportement analogue à basse température, passe par un minimum pour atteindre une région activée à plus haute température. L'interprétation de ces résultats est fondée sur le modèle du transport en présence de fluctuations de potentiel à moyenne portée [39]; on considère l'existence selon la direction du transport, d'une distribution aléatoire du potentiel électrique qui modifie localement l'énergie du bord de bande. Dans ces conditions, une partie des porteurs reste localisée dans des puits de potentiel de profondeur moyenne $\Gamma$ et ne peut contribuer au courant. Seule une proportion $n_{\mathrm{f}}$ du nombre total de porteurs dans la bande, est libre; les conductivités en obscurité et sous lumière s'écrivent :

$$
\sigma_{0}=q \mu n_{\mathrm{f}} \quad \text { et } \quad \Delta \sigma=\frac{\Delta n_{\mathrm{f}}}{\Delta n} q \mu \Delta n
$$

où $\Delta n$ est la densité de porteurs photoexcités et $n_{\mathrm{f}} \# n \times \exp (-\Gamma / k T)$. Ainsi donc, lorsque le taux d'injection demeure constant, tous les porteurs photoexcités participent à la conduction dans le cas d'un matériau dégénéré (soit $\Delta n_{\mathrm{f}} \# \Delta n$ ) et seulement une fraction lorsque le matériau devient non dégénéré $\left(\Delta n_{\mathrm{f}}<\Delta n\right)$; cette fraction tend vers 1 à haute température [39]. Ce comportement qualitatif explique donc l'allure de la photoconductivité observée (Fig. 3.3 ) et permet de déduire après ajustement les principaux paramètres du modèle $(\Gamma, n, \Delta n)$ [39].

3.2.2 Effet Hall. - Le coefficient de Hall et la mobilité de Hall de couches de type $\mathrm{P}$ de SOS ont été mesurés par Roux et Bielle-Daspet [41] (Fig. 3.4a et $b$ ). Les variations de la mobilité de Hall avec la température suggèrent que les processus de collision sur les impuretés et sur les dislocations sont plus importants pour les échantillons de faible épaisseur. Pour l'échantillon le plus épais $(2,4 \mu \mathrm{m}), \mu_{\mathrm{H}}$ varie sensiblement comme dans le cas du Si massif mais reste inférieur d'un facteur 2 aux valeurs de volume publiées par Morin et Maita [47]. Le coefficient de Hall, dont découle la concentration de trous, présente une région activée à basse température compatible avec l'énergie d'ionisation des accepteurs (bore) : $E_{\mathrm{a}}=45 \mathrm{meV}$. Ces résultats sont en accord avec ceux obtenus par d'autres auteurs $[48,49]$.

Robert et al. [39] ont étudié l'effet Hall sur du SOS de type $\mathbf{N}$ en fonction de la température. Leurs résultats (Fig. 3.5) montrent que le matériau possède un comportement dégénéré à basse température, qui vu la valeur de la mobilité (\# $130 \mathrm{~cm}^{2} / \mathrm{Vs}$ ), ne peut être attribué à une conduction par bande d'impuretés. L'énergie d'activation de l'ordre de $12 \mathrm{meV}$, trouvée dans la région à haute température, implique pour le substrat une valeur du dopage d'environ 3,5 $\times$ $10^{18} \mathrm{~cm}^{-3}$, valeur très supérieure au dopage présumé des couches, compte tenu de la dose d'implantation de phosphore $\left(\# 10^{16} \mathrm{~cm}^{-3}\right)$. Les auteurs en concluent que seule une faible épaisseur du film contribue à la conduction. La couche de transition située à l'interface $\mathrm{Si}-\mathrm{Al}_{2} \mathrm{O}_{3}$, qui doit être responsable de ce comportement pseudo-métallique, a une épaisseur estimée à $80 \AA$ [39], valeur en accord global avec celles obtenues par d'autres [38, 40, 50-52].

3.2.3 Magnétorésistance. - Les mesures de MR longitudinale et transversale à faible champ magnétique ont permis de révéler les contraintes et l'anisotropie des films SOS [27, 30, 31]. En exploitant la MR longitudinale sous champ magnétique intense, Cristoloveanu et al. [38] ont montré que le coefficient d'anisotropie $K$ de l'ensemble du film était voisin de 7 ; une analyse détaillée de ce résultat sera effectuée au $\S 3.3$.

3.2.4 Pouvoir thermoélectrique. - D'une façon générale, la caractérisation des semiconducteurs par effets de transport privilégie l'effet Hall par rapport au pouvoir thermoélectrique (PTE). Cependant, Ghibaudo et Kamarinos [40] ont souligné l'utilité du PTE dans le cas des matériaux transversalement inhomogènes; tel est le cas du SOS qui présente un caractère bicouche dû à la région de transition à l'interface $\mathrm{Si}-\mathrm{Al}_{2} \mathrm{O}_{3}[50,51]$.

Ghibaudo et al. ont étudié le PTE en fonction de la température sur des films de SOS de type $N[40,44]$. Les résultats obtenus (Fig. 3.6) montrent que si pour $T>200 \mathrm{~K}$, le PTE présente des valeurs compatibles avec le dopage moyen des couches $\left(10^{16}\right.$ à $7 \times$ $10^{17} \mathrm{~cm}^{-3}$ ), en revanche à basse température les variations du PTE sont surprenantes. En effet, dans le cas du Si massif de dopage analogue, on observe un accroissement du PTE en 1/T lorsque la température s'abaisse. Dans le cas du SOS le comportement est inversé ; les auteurs [40, 43-45] attribuent ce phénomène à la présence d'une couche dégénérée à l'interface $\mathrm{Si}-\mathrm{Al}_{2} \mathrm{O}_{3}$ responsable du caractère pseudométallique du PTE à basse température. Le modèle 

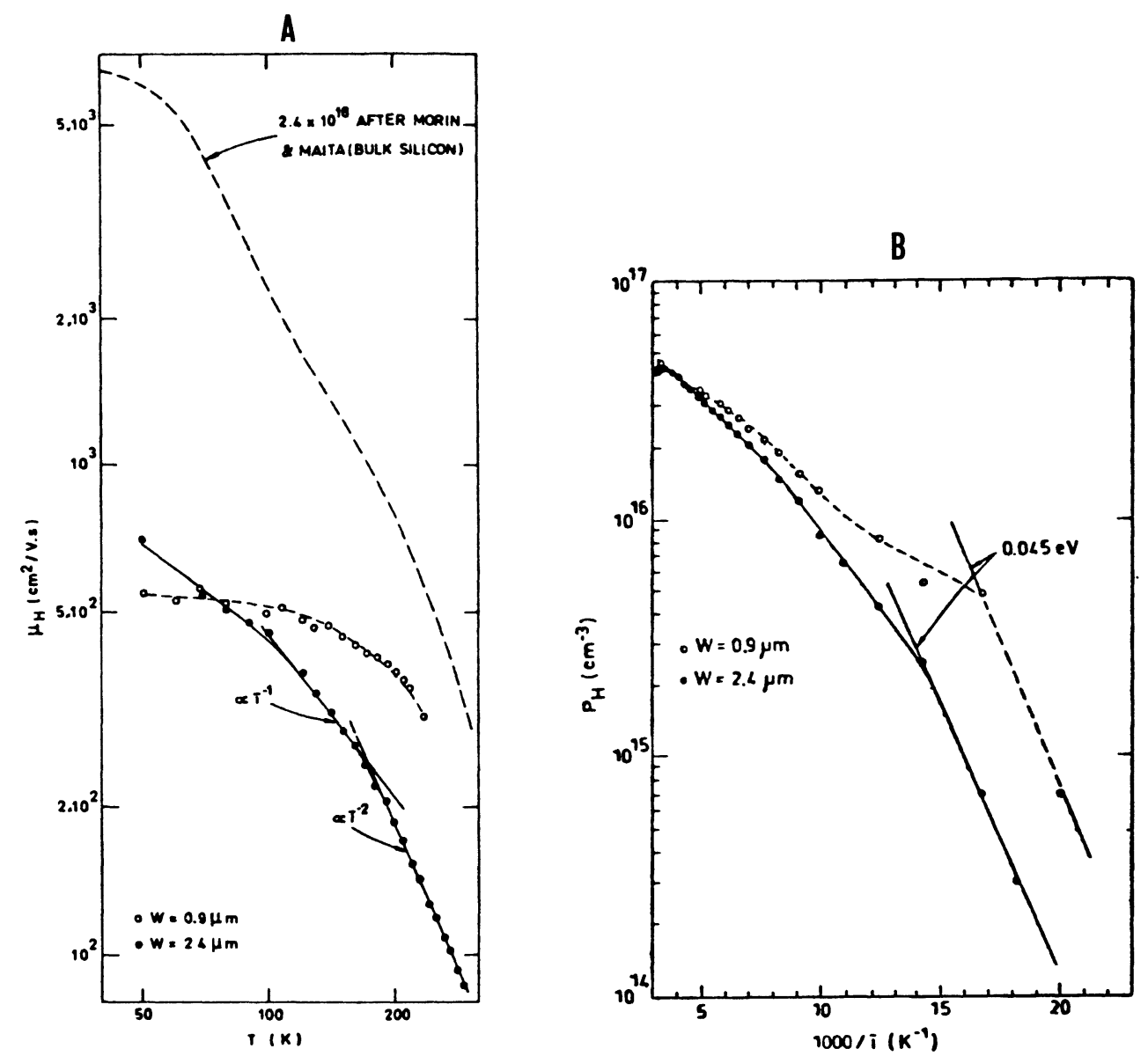

Fig. 3.4. - Variation de la mobilité de Hall $(a)$ et de la concentration $(b)$ des trous avec la température (d'après Roux et Bielle-Daspet [41]).

[Hall Mobility $(a)$ and hole concentration $(b)$ as a function of temperature (after Roux and Bielle-Daspet [41]).]
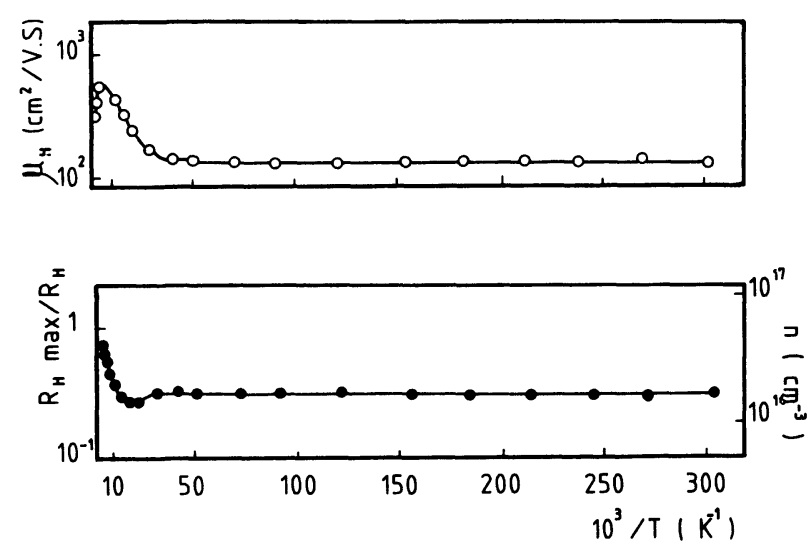

Fig. 3.5. - Variation de la mobilité de Hall et du coefficient de Hall dans n-SOS avec la température (d'après Robert et al. [39]).

[Hall mobility and Hall coefficient in n-SOS as a function of temperature (after Robert et al. [39]).]

bicouche leur permet de déduire des mesures de PTE et de conductivité, les principaux paramètres de transport dans la direction d'épitaxie, ainsi que l'épaisseur de la couche dégénérée. Ils trouvent que la partie supérieure du film de Si présente un caractère analogue au $\mathrm{Si}$ massif avec néanmoins une mobilité plus faible d'un facteur 2 , tandis que la couche de transition à l'interface $\mathrm{Si}-\mathrm{Al}_{2} \mathrm{O}_{3}$, de nature dégénérée sur une épaisseur de 100 à $150 \AA$, possède une forte densité de porteurs $\left(\# 10^{19}\right.$ à $10^{20} \mathrm{~cm}^{-3}$ ) dont la mobilité n'excède pas quelques dizaines $\mathrm{de}^{\mathrm{cm}^{2}} / \mathrm{Vs}$ $[40,44]$.

Des résultats complémentaires obtenus sur des couches de SOS de plus fort dopage $\left(\# 10^{19} \mathrm{~cm}^{-3}\right.$ ) $[45,53]$ ont montré la nécessité de prendre en compte non seulement le caractère bicouche du SOS mais aussi la présence de fluctuations de potentiel longitudinales dans le plan d'épitaxie rejoignant ainsi l'analyse de Robert et al. [39]. En outre, la couche de transition dégénérée à l'interface $\mathrm{Si}_{-} \mathrm{Al}_{2} \mathrm{O}_{3}$ doit plus vraisemblablement trouver son origine dans la forte densité de charges fixes $\left(\# 10^{12} \mathrm{~cm}^{-2}\right)$ dues à la proximité du saphir [45]. De plus les variations anormales du PTE et de la conductivité à basse température, obtenues sur des films SOS dégénérés, ont permis de mettre en évidence l'existence d'un seuil de mobilité caractéristique d'une forte localisation [53]. 


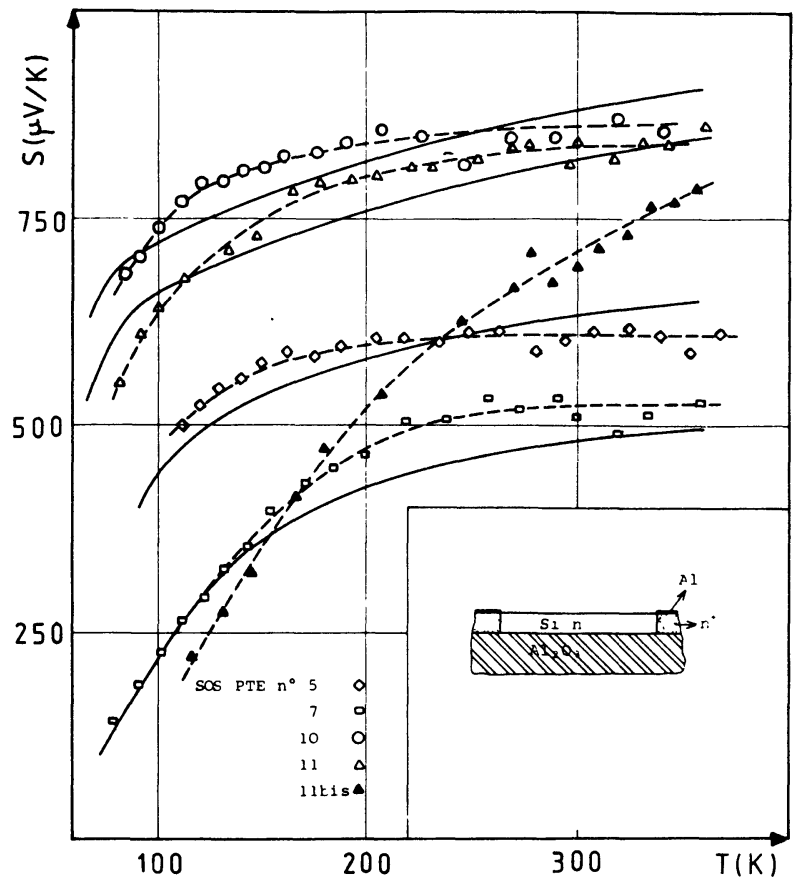

Fig. 3.6. - Variation du pouvoir thermoélectrique avec la température : en pointillés courbes expérimentales, en traits pleins courbes issues du modèle bicouche (d'après Ghibaudo et Kamarinos [40]).

[Thermopower as a function of temperature : experimental curves (dashed lines) and theoretical curves (full lines) (after Ghibaudo and Kamarinos [40]).]

L'ensemble des résultats obtenus par les mesures globales des propriétés de transport fait ressortir [30-54] (i) la détérioration de la qualité électrique des couches SOS par rapport au Si massif, (ii) l'affaiblissement de la mobilité dû aux contraintes et aux processus de collisions supplémentaires et (iii) la présence d'inhomogénéités spécifiques tant longitudinales (fluctuations de potentiel) que transversales (couche de transition à l'interface $\mathrm{Si}^{-} \mathrm{Al}_{2} \mathrm{O}_{3}$ ). Dans ces conditions la détermination des profils exacts des paramètres de transport est de première importance pour une description plus précise du matériau.

\subsection{ProfiLS DES COEFFICIENTS DE TRANSPORT DANS LE SOS.}

3.3.1 Fondements théoriques et principes de mesure. Une méthode directe de caractérisation des inhomogénéités transversales consiste à combiner effets magnétoélectriques et effet de champ [36, 46, 55]. Ainsi, dans une structure de type MOS-Hall à déplétion profonde (cf. encart Fig. 3.7), la variation de la tension de grille $V_{\mathrm{g}}$ rend possible la modulation de l'épaisseur $W$ de la couche active du film. Lorsque la tension de grille est incrémentée de $\Delta V_{\mathrm{g}}$, l'épaisseur de la région désertée par les porteurs se trouve modifiée de $\Delta W$; on détermine alors par différenciation la contribution au phénomène considéré d'une couche active d'épais- seur infinitésimale $\Delta W$ située à la frontière de la région de désertion. Pour chaque coefficient de transport $A$ il devient donc nécessaire de bien distinguer sa valeur ponctuelle, notée $A^{*}(z)$, de sa valeur moyenne $A\left(V_{\mathrm{g}}\right)$ mesurée expérimentalement dans la zone active. La correspondance entre tension de grille et profondeur de déplétion se déduit d'après des mesures de capacité MOS.

Sur le plan théorique, l'analyse des effets de transport dans les matériaux inhomogènes a comme point de départ le tenseur de conductivité, dont les éléments sont fonction des coordonnées géométriques. Le cas du Si de type $n$, présentant des inhomogénéités unidirectionnelles, a été rigoureusement traité par Cristoloveanu et al. [38]; ce modèle a été ensuite transposé au cas du SOS [36]. On montre que les coefficients galvanomagnétiques dans un matériau inhomogène ne sont pas de simples moyennes des coefficients correspondant au matériau homogène. Il est fondamental de considérer comme fonctions de $z$, non seulement les composantes $\sigma_{\alpha \beta}^{*}(z)$ du tenseur de conductivité (Eq. 3.1), mais aussi les équations propres (conservation du courant, équations de Maxwell) au phénomène étudié; ce sont les conditions aux limites qui guideront ensuite le choix des expressions devant être moyennées.

3.3.2 Profils expérimentaux de conductivité, de magnétorésistance et d'effet Hall. - L'intérêt de la mesure de la capacité des structures MOS/SOS est relatif, entre autres, à la détermination de l'épaisseur $W_{\mathrm{d}}$ de la zone désertée et du profil d'impuretés ionisées, ainsi qu'à l'estimation du profil de mobilité. Durant les mesures de capacité, les effets dus aux états d'interfaces et à la résistance série de la couche active ont été minimisés par le choix de la fréquence de mesure [32]. L'épaisseur $W_{\mathrm{d}}$ est donnée par $[55,56]$ :

$$
W_{\mathrm{d}}=\varepsilon l_{X} l_{Y}\left(C^{-1}\left(V_{\mathrm{g}}\right)-C_{0 x}^{-1}\right)
$$

où $\varepsilon$ est la permittivité $\mathrm{de} \mathrm{Si}, l_{X}, l_{Y}$ sont la longueur et la largeur de la structure (Fig. $3.1 b$ et 3.7 ), $C_{0 x}$ la capacité de l'oxyde. La courbe $W_{\mathrm{d}}\left(V_{\mathrm{g}}\right)$ est tracée en tenant compte de la tension de "bande plate" $(-1,1 \mathrm{~V})$ déduite d'après les courbes $\left(C_{0 x} / C\right)^{2} \propto V_{\mathrm{g}}$ [32]. La résistance $R_{B}$ de la couche active (dans le cas général d'un champ magnétique $B$ ) s'exprime en fonction de la conductivité électrique ponctuelle $\sigma_{B}^{*}$ :

$$
R_{B}(V)=\frac{l_{X}}{l_{Y}}\left[\int_{0}^{W} \sigma_{B}^{*}(z) \mathrm{d} z\right]^{-1}
$$

en dérivant on accède au profil de conductivité :

$$
\sigma_{B}^{*}\left(z=W\left(V_{\mathrm{g}}\right)\right)=\frac{l_{X}}{l_{Y}} \frac{\mathrm{d}\left(R_{B}^{-1}\right)}{\mathrm{d} V_{\mathrm{g}}} \frac{\mathrm{d} V_{\mathrm{g}}}{\mathrm{d} W} .
$$

Notons qu'il s'agit d'un profil expérimental, ne dépendant d'aucune hypothèse théorique exceptée la relation classique (3.5), utile pour obtenir $V_{\mathrm{g}}(W)$; de plus, le terme $\mathrm{d} V_{\mathrm{g}} / \mathrm{d} W$ disparaît lors de la détermi- 


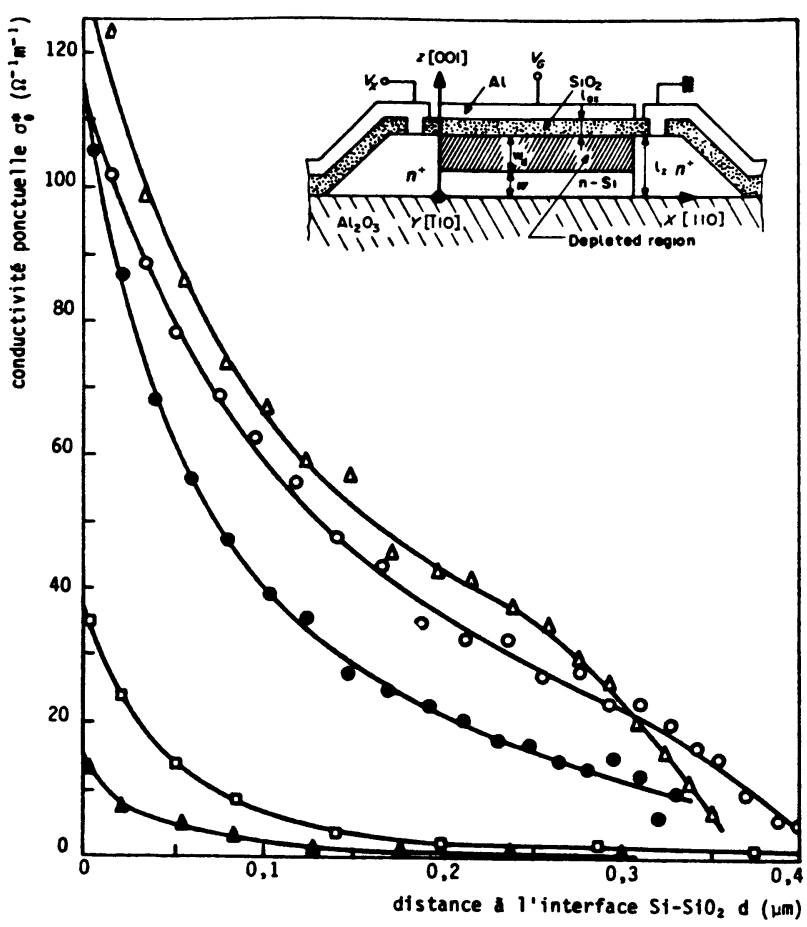

Fig. 3.7. - Profil de conductivité électrique $\sigma_{0}^{*}(z)$ en fonction de la distance à l'interface $\mathrm{Si}_{-} \mathrm{SiO}_{2}$. En insertion : structure et orientation cristallographique de l'échantillon MOS-Hall/SOS de dimensions $1200 \times 800 \times 0,65 \mu^{3}$ (cf. Fig. 3.1) et de dopage $\mathrm{N}$ entre $10^{15}$ et $10^{16} \mathrm{~cm}^{-3}$ (d'après Lee et al. [36]).

[Electrical conductivity profile $\sigma_{0}^{*}(z)$ as a function of distance from the $\mathrm{Si}_{-} \mathrm{SiO}_{2}$ interface. Inserted is the structure and the cristallographic orientation of MOS-Hall/SOS samples with dimensions $1200 \times 800 \times 0.65 \mu \mathrm{m}^{3}$ (cf. Fig. 3.1) and $\mathrm{N}$-type doping in the range $10^{15}$ to $10^{16} \mathrm{~cm}^{-3}$ (after Lee et al. [36]).]

nation du coefficient de magnétorésistance $\rho_{B}^{*} / \rho_{0}^{*}=$ $\sigma_{0}^{*} / \sigma_{B}^{*}$.

La figure 3.7 illustre la dégradation dramatique de la conductivité $\sigma_{0}^{*}$ lorsqu'on s'approche de l'interface
$\mathrm{Si}-\mathrm{Al}_{2} \mathrm{O}_{3}$ [36] et confirme ainsi l'aspect fortement inhomogène du transport dans le SOS.

En figure 3.8 sont reportés les profils de magnétorésistance obtenus pour la première fois par Lee et al. [36, 57]. L'importante valeur de la MR longitudinale reflète, d'une part, la forte anisotropie du SOS (et du $\mathrm{Si}$ ) et confirme, d'autre part, l'existence de contraintes d'interface, qui accentuent l'effet (cf. § 3.1.2, éq. 3.4). La MR longitudinale diminue sensiblement non seulement à proximité de l'interface $\mathrm{Si}-\mathrm{Al}_{2} \mathrm{O}_{3}$ (en raison des collisions électroniques sur la surface) mais surtout dans le volume, phénomène attribué essentiellement à la réduction de la mobilité [36]. Au contraire, la MR transversale tend à augmenter pour des distances supérieures à $0,25 \mu \mathrm{m}$, fait qui suggère déjà une modification qualitative du processus de collisions.

La mobilité ponctuelle de Hall $\mu_{\mathrm{H}}^{*}(z)$ se déduit d'après les valeurs de la mobilité moyenne $\left\langle\mu_{\mathrm{H}}\right\rangle\left(V_{\mathrm{g}}\right)$, mesurées dans la région active (et à faible champ magnétique) [37] :

$$
\begin{aligned}
\left\langle\mu_{\mathrm{H}}\right\rangle\left(V_{\mathrm{g}}\right)=\left[\int_{0}^{W} \mu_{\mathrm{H}}^{*}(z)\right. & \left.\sigma_{0}^{*}(z) \mathrm{d} z\right] \times \\
\times & {\left[\int_{0}^{W} \sigma_{0}^{*}(z) \mathrm{d} z\right]^{-1} . }
\end{aligned}
$$

En dérivant $[36,37]$ :

$$
\mu_{\mathrm{H}}^{*}(z=W)=\left\langle\mu_{\mathrm{H}}\right\rangle\left(V_{\mathrm{g}}\right)-R_{0}\left(V_{\mathrm{g}}\right) \frac{\mathrm{d}\left\langle\mu_{\mathrm{H}}\right\rangle}{\mathrm{d} R_{0}} .
$$

Le profil présenté en figure 3.9 montre que $\mu_{\mathbf{H}}^{*}$ diminue d'un facteur 2 sur environ $0,4 \mu \mathrm{m}$ à l'intérieur du film. Même loin de l'interface $\mathrm{Si}-\mathrm{Al}_{2} \mathrm{O}_{3}$, la mobilité de Hall $\left(600 \mathrm{~cm}^{2} / \mathrm{Vs}\right)$ n'atteint que 30 à $50 \%$ de la valeur usuelle pour le Si massif; ce résultat est en très bon accord avec les prévisions du modèle théorique incluant l'effet des contraintes (cf. § 3.1, Eq. 3.3).
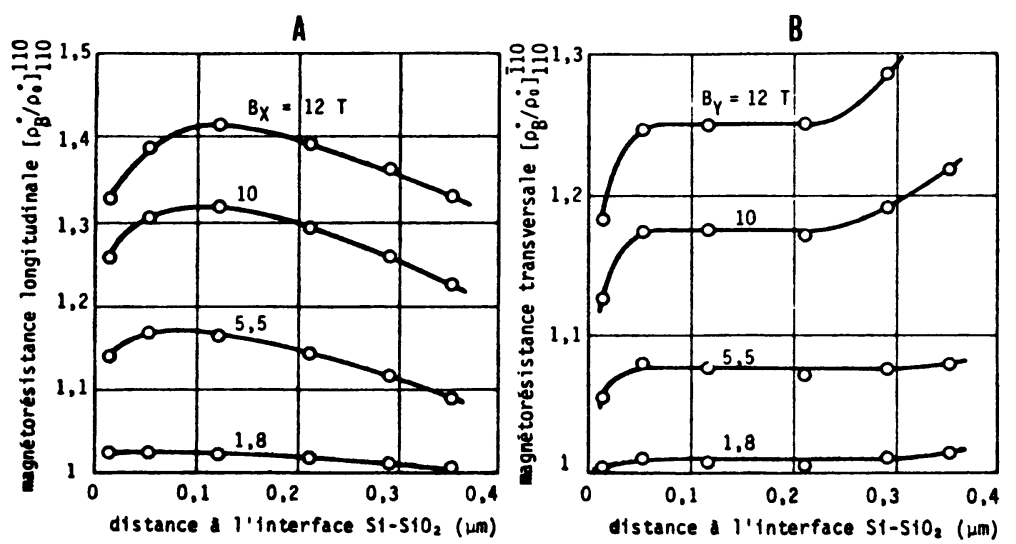

Fig. 3.8. - Distribution expérimentale du coefficient ponctuel de magnétorésistance longitudinale $(a)$ et transversale $(b)$ (d'après Lee et al. [36]).

[Experimental distribution of longitudinal $(a)$ and transverse $(b)$ magnetoresistance coefficients (after Lee et al. [36]).] 
3.3.3 Détermination des profils de mobilité, d'anisotropie et de concentration. - Une première méthode classique $[55,56]$ de détermination du profil de la mobilité de dérive consiste à déduire des courbes $C\left(V_{\mathrm{g}}\right)$ d'une structure MOS les valeurs ponctuelles de la concentration :

$$
n(z=W)=\frac{C\left(V_{\mathrm{g}}\right)}{e l_{X} l_{Y}} \frac{\mathrm{d} V_{\mathrm{g}}}{\mathrm{d} W}
$$

et donc de la mobilité :

$$
\mu_{0}^{*}(z)=-\sigma_{0}^{*}(z) / e n^{*}(z) .
$$

Cependant, un examen approfondi tend à prouver que la variation de mobilité ainsi obtenue (Fig. 3.9) est peu crédible [36]; il est en effet impossible, à partir de ce profil, de retrouver théoriquement les courbes $\mu_{\mathrm{H}}^{*} / \mu_{0}^{*}$ (voir aussi $\S 3.3 .4$ ).

La seconde méthode, mise au point récemment $[36,37,58]$ se fonde sur le traitement numérique des courbes de MR longitudinale. On utilise le formalisme présenté au $\S 3.1$, en considérant, cette fois, que les coefficients de transport sont inhomogènes. Comme la MR longitudinale est essentiellement gouvernée par l'anisotropie du matériau et dépend peu des mécanismes collisionnels [38] on peut adopter l'hypothèse de porteurs monocinétiques (d'autant plus satisfaisante que la gamme de champs magnétiques est étendue [37]); le coefficient ponctuel de MR longitudinale est alors donné par :

$$
\begin{aligned}
{\left[\frac{\rho_{B}^{*}(z)}{\rho_{0}^{*}(z)}\right]_{110}^{110}=} & {\left[1+\frac{2 K^{*}}{K^{*}+1}\left(\mu_{0}^{*} B\right)^{2}\right] \times } \\
& \times\left[1+\frac{8 K^{*}}{\left(K^{*}+1\right)^{3}}\left(\mu_{0}^{*} B\right)^{2}\right]^{-1}
\end{aligned}
$$

expression équivalente, pour de très forts champs magnétiques à l'équation "inhomogène " 3.4. La relation 3.12 fournit, par comparaison aux courbes expérimentales $\rho_{B}^{*} / \rho_{0}^{*}(B)$ (duales à celles de la Fig. $3.8 a)$ les valeurs ponctuelles de $K^{*}(z)$ et de $\mu_{0}^{*}(z)$.

La figure 3.9 montre que la mobilité de dérive des électrons décroît quasi linéairement avec la distance à l'interface $\mathrm{Si}_{-} \mathrm{SiO}_{2}$, le taux de dégradation étant de 15 à $20 \%$ par $1000 \AA$ [36]. Par ailleurs, l'écart entre les mobilités de Hall et de dérive, déterminées expérimentalement, représente un excellent support pour le modèle des 4 vallées iso-énergétiques (cf. $\S 3.1$ ) adopté pour la bande de conduction du SOS, modèle qui laissait prévoir un rapport $\mu_{\mathrm{H}}^{*} / \mu_{0}^{*}$ de l'ordre de $60 \%$. La figure 3.9 montre aussi qu'au voisinage de l'interface $\mathrm{Si}_{-} \mathrm{SiO}_{2}$ la mobilité atteint $1100 \mathrm{~cm}^{2} / \mathrm{Vs}$, ce qui (correspondant en absence des contraintes à $1400 \mathrm{~cm}^{2} / \mathrm{Vs}$ [36]) reflète la qualité cristalline très convenable de la partie supérieure du film SOS.

Le coefficient d'anisotropie de masse effective et de temps de relaxation $K^{*}(z)$ se présente comme une

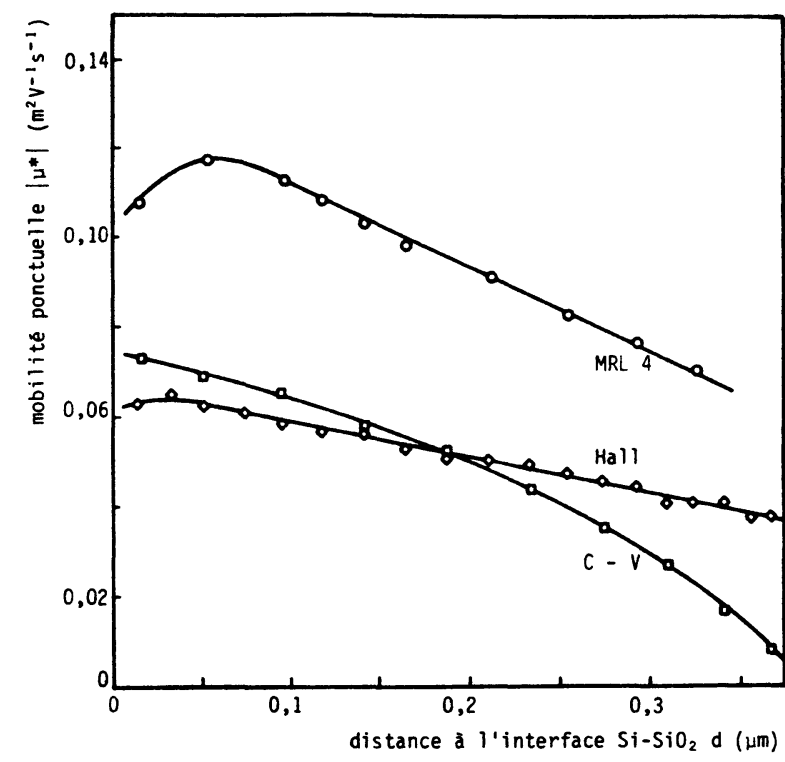

Fig. 3.9. - Profils de la mobilité des électrons dans le SOS, déduits des mesures d'effet Hall $\left(\mu_{\mathrm{H}}^{*}\right)$ de magnétorésistance longitudinale (MRL) et de capacité $(\mathrm{C}-\mathrm{V})$ (d'après Lee et al. [36]).

[Electron mobility profiles in SOS obtained by Hall measurements $\left(\mu_{\mathbf{H}}^{*}\right)$, longitudinal magnetoresistance (MRL) and capacitance (C-V) (after Lee et al. [36]).]

fonction légèrement croissante avec la distance à l'interface $\mathrm{Si}_{-} \mathrm{SiO}_{2}$ [36]. Sa valeur moyenne $\left\langle K^{*}\right\rangle \# 5$ à 6 s'avère être en bon accord, d'une part, avec les résultats de déterminations globales $(K \# 5$ [30] et $K$ \# 7 [38]) et, d'autre part, avec la valeur usuelle du $\mathrm{Si}$ massif [34].

En se référant aux profils des mobilités et de l'anisotropie, Lee et al. [36] déterminent également la variation du coefficient $r^{*}(z)=\overline{\tau^{2}} / \bar{\tau}^{2}$ qui définit le processus de relaxation (cf. Eq. $3.2 b$ et $3.3 a$ ) :

$$
r^{*}(z)=\frac{\left(K^{*}+1\right)^{2}}{4 K^{*}} \frac{\mu_{\mathrm{H}}^{*}}{\mu_{0}^{*}} .
$$

Le profil obtenu montre près de l'interface $\mathrm{Si}-\mathrm{SiO}_{2}$ une prédominance de collisions avec les phonons acoustiques $\left(r^{*} \# 3 \pi / 8\right)$ et, plus loin, une accentuation du rôle des impuretés ionisées ( $r^{*}$ augmente). Cette observation est parfaitement compatible avec l'accroissement du coefficient ponctuel de MR transversale, lorsque la distance à l'interface $\mathrm{Si}-\mathrm{SiO}_{2}$ devient supérieure à $0,25 \mu \mathrm{m}$.

Le profil de la concentration de porteurs (Fig. 3.11), extrait des courbes de conductivité (Fig. 3.7) et de mobilité de dérive (Fig. 3.9), présente une allure quasi exponentielle $[36,59]$. Nous verrons au paragraphe suivant que la discussion des problèmes relatifs à la concentration des porteurs et aux mécanismes collisionnels est largement facilitée par les mesures à température variable. 
3.3.4 Influence de la température sur les profils de mobilité et de concentration. - Des mesures magnétoélectriques contrôlées par effet de champ ont été réalisées entre 77 et $300 \mathrm{~K}[32,58-60]$. La figure 3.10 présente l'influence de la température sur la conductivité électrique et sur la mobilité de Hall à différentes profondeurs dans le film. Pour des profondeurs supérieures à $1000 \AA \AA$ on remarque que la conductivité diminue régulièrement lorsque la température décroît, par suite des variations de la concentration et de la mobilité des électrons. On attribue la partie la plus abrupte des courbes $(150 \mathrm{~K}<T<300 \mathrm{~K})$ à l'ionisation des impuretés non intentionnelles [32]; comme la pente devient plus forte avec la profondeur il résulte que le rôle de ces impuretés s'accentue en approchant du substrat. Pour $d<1000 \AA$ la conductivité est peu modifiée entre 150 et $300 \mathrm{~K}$, ce qui souligne la faible importance, dans la couche supérieure, des impuretés non intentionnelles.

Le comportement de la mobilité de Hall est très complexe car il dépend de la profondeur et de la gamme de température. Pour des températures supérieures à $120 \mathrm{~K}$, les collisions avec les phonons acoustiques $\left(\mu_{\mathrm{H}} \propto T^{-1,5}\right)$ prédominent uniquement au voisinage de l'interface $\mathrm{Si}_{-} \mathrm{SiO}_{2}$, ce qui traduit une qualité cristalline de la couche supérieure comparable au Si massif (où, au-dessus de $100 \mathrm{~K}, \mu_{\mathrm{H}}$ décroît également avec T). En s'approchant de l'interface $\mathrm{Si}-\mathrm{Al}_{2} \mathrm{O}_{3}$, la pente des courbes s'inverse, sous l'influence des collisions avec les impuretés ionisées $\left(\mu_{\mathrm{H}} \propto T^{1,5}\right)$; à une distance de $3000 \AA$ du substrat ces collisions prédominent même à la température ambiante.

Autour de la température "critique" de $120 \mathrm{~K}$, la mobilité ponctuelle de Hall présente un minimum quelle que soit la profondeur dans le film. Cette pro- priété confirme $[32,60]$ la participation des impuretés non intentionnelles provenant du substrat $[4,24,25]$ qui (en raison d'un niveau énergétique légèrement plus profond que celui du phosphore) semblent être ionisées au-dessus de $120 \mathrm{~K}$; elles se présentent sous forme de complexes de grandes sections efficaces et jouent un rôle important comme centres de collisions électroniques. L'existence d'une température critique, associée aux impuretés non intentionnelles, a été également observée par Ipri et Zemel [49]; leur résultat, $T_{c}=210 \mathrm{~K}$, est différent par suite de la technologie utilisée et surtout parce qu'ils se sont fondés sur la variation en température de la mobilité moyenne de la couche active.

Pour des températures inférieures à $120 \mathrm{~K}$ l'allure des courbes est approximativement en $T^{-0,5}$ quelle que soit la distance; cette variation est attribuée à un processus mixte de collisions, englobant l'effet des dislocations neutres $(T)$, de charges d'espace $\left(T^{-0,5}\right)$, des phonons $\left(T^{-1,5}\right)$, des charges cylindriques $(T)$, etc... $[32,60]$. De plus, l'examen de la figure $3.10 b$ montre qu'à basse température $(80 \mathrm{~K}<T<200 \mathrm{~K})$ la mobilité se dégrade d'un facteur 5 sur une distance d'environ $0,35 \mu \mathrm{m}$, alors qu'à $300 \mathrm{~K}$ la balance entre collisions acoustiques et coulombiennes limite la décroissance à environ $50 \%$.

Le comportement des impuretés non intentionnelles, élément clef dans la discussion des profils de conductivité et de mobilité, a été révélé aussi par les mesures capacitives [59]. Ainsi, lorsqu'on suppose que la densité des accepteurs et des défauts cristallins chargés négativement est négligeable par rapport à celle des donneurs dans le film n-SOS, l'équation 3.10 fournit le nombre d'impuretés ionisées $N_{\mathrm{D}}^{*}(z)$, correspondant au nombre total d'électrons dans la bande de conduc-
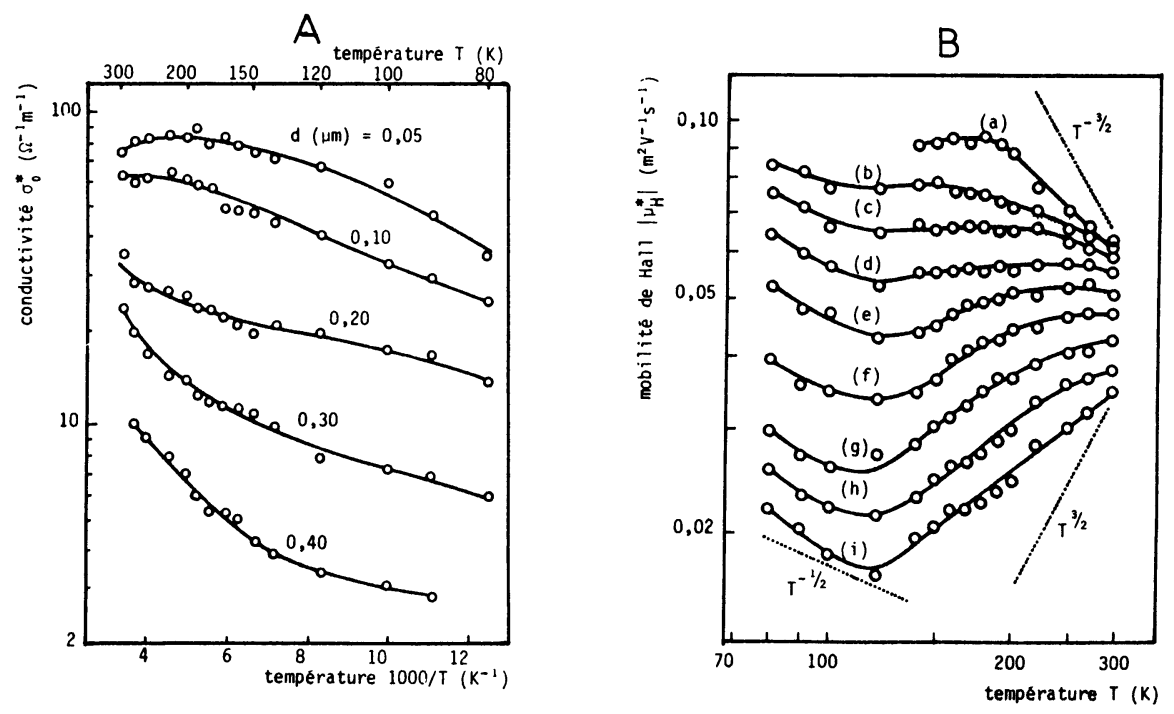

Fig. 3.10. - Variation de la conductivité ponctuelle $\sigma_{0}^{*}(z)$ (Fig. $\left.a\right)$ et de la mobilité de Hall ponctuelle $\mu_{\mathrm{H}}^{*}(z)$ (Fig. $\left.b\right)$ en fonction de la température pour plusieurs distances $d(\mu \mathrm{m})$ à l'interface $\mathrm{Si}_{-} \mathrm{SiO}_{2}$ incrémentées de $500 \AA$ (d'après Lee [32, 60])

[Electrical conductivity $\sigma_{0}^{*}(z)$ (Fig. $a$ ) and Hall mobility $\mu_{\mathrm{H}}^{*}(z)$ (Fig. $\left.b\right)$ versus temperature at several depths $d(\mu \mathrm{m})$, increased by $500 \AA$ steps, from the $\mathrm{Si}_{-} \mathrm{SiO}_{2}$ interface (after Lee $\left.[32,60]\right)$.] 
tion $n_{\mathrm{tot}}^{*}(z)$. La figure 3.11 montre qu'au voisinage de l'interface $\mathrm{Si}_{-} \mathrm{SiO}_{2}$ la densité des impuretés augmente peu avec la température; ceci révèle le rôle prédominant des impuretés dopées intentionnellement (phosphore) dont l'ionisation se fait à basse température $(T<100 \mathrm{~K})$. Au contraire près de l'interface $\mathrm{Si}-\mathrm{Al}_{2} \mathrm{O}_{3}$, la densité $N_{\mathrm{D}}^{*}(z)$ s'accroît fortement lorsque la température s'élève de 160 à $300 \mathrm{~K}$, confirmant l'ionisation progressive des impuretés " autodopées " au-dessus de $160 \mathrm{~K}$. En ce qui concerne le profil de concentration, il est à noter qu'à la température ordinaire la densité des impuretés autodopées augmente très nettement pour des distances à l'interface film-substrat inférieures à $0,3 \mu \mathrm{m}$. Les résultats à $90 \mathrm{~K}$ montrent que la densité des impuretés intentionnelles décroît de façon monotone (d'un facteur 3) avec la profondeur sur 0,4 $\mu \mathrm{m}$ [59].

La figure 3.11 contient également le profil de porteurs $n^{*}(z)$ à $296 \mathrm{~K}$, qui résulte du traitement des profils de conductivité $\sigma_{0}^{*}(z)$ (Fig. 3.7) et de mobilité de dérive $\mu_{0}^{*}(z)$ (courbe MRL, Fig. 3.9) selon l'équation 3.11. Cette courbe provient donc de la mesure des effets de transport et correspond par conséquent à la densité

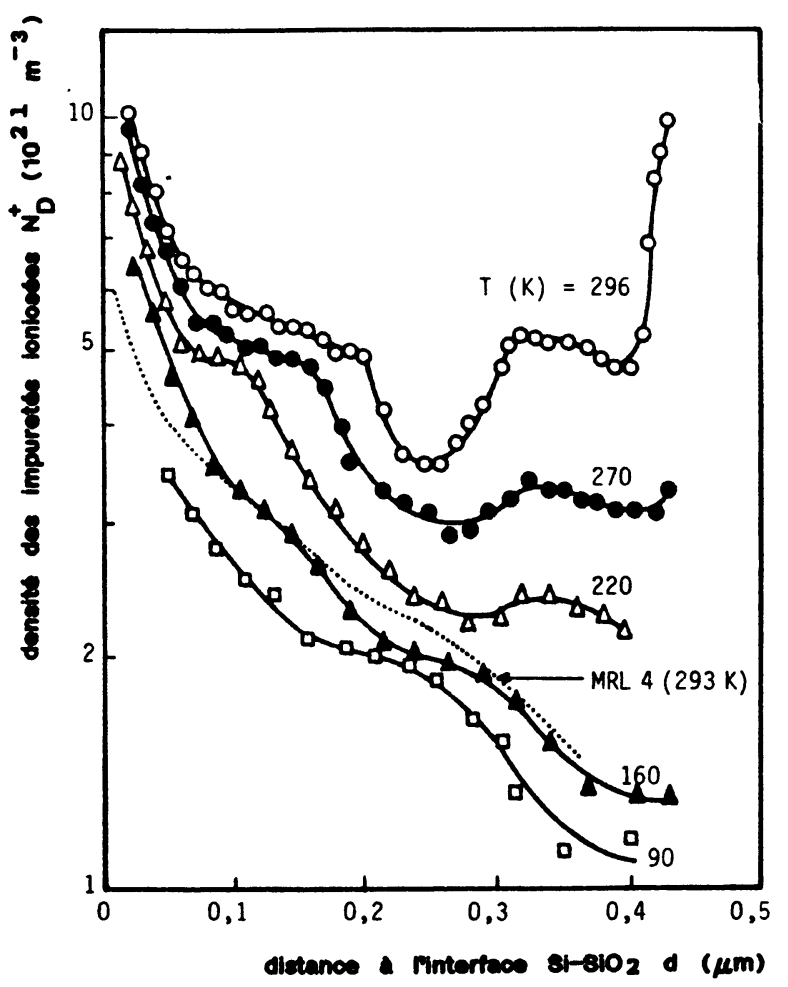

Fig. 3.11. - Profils des impuretés donatrices ionisées $\mathrm{N}_{\mathrm{d}}^{+}(z)$ pour plusieurs températures (déduits des mesures capacitives). La courbe pointillée montre la densité des porteurs (électrons effectivement libres) déduite des mesures de magnétorésistance longitudinale et de conductivité à $293 \mathrm{~K}$ (cf. Fig. 3.7) (d'après Lee et al. [59]).

[Ionized donor impurity profiles $\mathrm{N}_{\mathrm{d}}^{+}(z)$ for several temperatures obtained by capacitance measurements. The carrier density (free electrons) deduced from longitudinal magnetoresistance and conductivity data at $293 \mathrm{~K}$ (cf. Fig. 3.7), is shown by the broken line (after Lee et al. [59]).] des électrons effectivement libres [59]. Rappelons que la courbe relevée à $296 \mathrm{~K}$ des mesures capacitives représente la densité totale d'électrons $n_{\mathrm{tot}}^{*}(z)$. La différence entre ces deux profils montre clairement qu'une partie des électrons ne participe pas au transport du courant et sont donc " localisés ». Les auteurs interprètent cette localisation à l'aide du modèle des fluctuations de potentiel proposé par Robert et al. (cf. § 3.2) [39]. La profondeur $\Gamma(z)$ du puits de potentiel, correspondant à la fluctuation moyenne du bord de la bande de conduction, a été estimée d'après la relation [59] :

$$
\Gamma(z)=k T \log \frac{n_{\mathrm{tot}}^{*}(z)}{n^{*}(z)} .
$$

La profondeur moyenne des puits est de l'ordre de $25 \mathrm{meV}$, en bon accord avec les résultats de Robert [39]. De plus, $\Gamma(z)$ (ou le rapport $n_{\text {tot }}^{*} / n^{*}$, cf. Fig. 3.11) augmente sensiblement pour des distances à l'interface Si-SiO ${ }_{2}$ supérieures à $0,2 \mu \mathrm{m}$ [59], fait qui illustre la détérioration de la qualité cristalline du film et peut être relié à la présence des impuretés non intentionnelles. Par ailleurs, l'écart entre $n_{\text {tot }}^{*}$ et $n^{*}$ explique pourquoi le profil de la mobilité de dérive $\mu_{0}^{*}$ déduit des mesures capacitives était si différent de celui déduit de la magnétorésistance (Fig. 3.9) et incompatible avec d'autres résultats de transport $[36,37]$.

3.4 PropriÉtÉS DES INTERFACES. - L'analyse des propriétés électriques des interfaces $\mathrm{Si}_{-} \mathrm{SiO}_{2}$ et $\mathrm{Si}$ $\mathrm{Al}_{2} \mathrm{O}_{3}$ est de première importance pour la détermination des performances des dispositifs MOS réalisés sur SOS. Si l'interface supérieure $\mathrm{Si}_{-} \mathrm{SiO}_{2}$ régit, en grande partie, le fonctionnement des TMOS et des capacités MOS, il n'en reste pas moins que la plupart des limitations de la technologie MOS/SOS est imputable à la mauvaise qualité de l'interface inférieure $\mathrm{Si}-\mathrm{Al}_{2} \mathrm{O}_{3}$ : courants de fuite importants [61], déformation des caractéristiques $I(V)$ des TMOS à forte tension drain-source par effet « kink » dû à la présence de pièges dans la couche de $\mathrm{Si}$ [62] ou à l'autopolarisation du substrat $[63,64]$...

L'étude des paramètres (mobilité des porteurs, concentration de charges fixes et de pièges, profil et densité d'états de surface) de l'interface $\mathrm{Si}_{-} \mathrm{SiO}_{2}$ est naturellement plus aisée que celle de l'interface $\mathrm{Si}-\mathrm{Al}_{2} \mathrm{O}_{3}$; en revanche l'interface $\mathrm{Si}-\mathrm{Al}_{2} \mathrm{O}_{3}$ peut être étudiée, d'une part, de façon indirecte par ces conséquences sur les caractéristiques électriques de dispositifs conventionnels réalisés face avant (métal$\mathrm{Si}-\mathrm{SiO}_{2}$ ) et, d'autre part, de façon directe grâce à des dispositifs spécifiques réalisés face arrière (métal$\mathrm{Al}_{2} \mathrm{O}_{3}-\mathrm{Si}$ ).

3.4.1 Interface $\mathrm{Si}_{-} \mathrm{SiO}_{2}$. - La mobilité des porteurs en surface est le paramètre essentiel pour l'évaluation de la qualité de l'interface. Une valeur trop faible limite le temps de transit des porteurs dans le canal d'un TMOS et altère leurs performances dynamiques; elle traduit par ailleurs, un mauvais " état de surface" 
résultant d'un processus d'oxydation de grille défectueux.

Les études menées au LETI [42, 65-68] montrent que les mobilités de surface déduites des caractéristiques $I_{\mathrm{d}}\left(V_{\mathrm{g}}\right)$ de TMOS en forte inversion sont typiquement :

$$
\begin{aligned}
& \left.\mu_{\mathrm{n}}=400 \text { à } 600 \mathrm{~cm}^{2} / \mathrm{Vs} \text { pour les électrons (canal } \mathrm{N}\right) . \\
& \left.\mu_{\mathrm{p}}=150 \text { à } 250 \mathrm{~cm}^{2} / \text { Vs pour les trous (canal } \mathrm{P}\right) .
\end{aligned}
$$

Ces valeurs montrent peu de différence entre le SOS et le $\mathrm{Si}$ massif et sont en accord avec celles généralement rencontrées dans la littérature [25].

Gris et Bello [42] ont constaté que l'épaisseur de la couche de Si épitaxié a une faible influence sur la mobilité de surface (Fig. 3.12). Ce résultat confirme que les phénomènes qui limitent la valeur de la mobilité dans une couche d'inversion sont étroitement corrélés à la qualité de l'interface et très peu aux propriétés du volume sous-jacent. Néanmoins aux faibles épaisseurs de Si épitaxié $(0,1-0,2 \mu \mathrm{m})$ le comportement de la mobilité de surface demeure surprenant : diminution escomptée de la mobilité $\mu_{\mathrm{p}}$ dans le canal $\mathbf{P}$ (dégradation du volume sous-jacent trop importante pour maintenir la qualité de l'interface), mais augmentation de $\mu_{\mathrm{n}}$ dans le canal $\mathrm{N}$. Il est vraisemblable compte tenu des remarques de Kawaji et al. [69] et de Englert et al. [70], que l'anisotropie des contraintes film-substrat, d'autant plus importante que l'épaisseur du film est faible, soit responsable de ce phénomène. La structure de la bande de conduction à deux dimensions du $\mathrm{Si}(100)$ favorise dans ces conditions l'existence des minimas énergétiques de plus faible masse effective dans lesquels la mobilité est plus importante.

Les processus qui limitent la valeur de la mobilité en surface (inversion ou accumulation) sont régis par les mécanismes prédominants de collision des porteurs d'un gaz bidimensionnel [71] : diffusion coulombienne due aux charges d'interface, diffusion de rugosité de surface et diffusion par les phonons acoustiques de

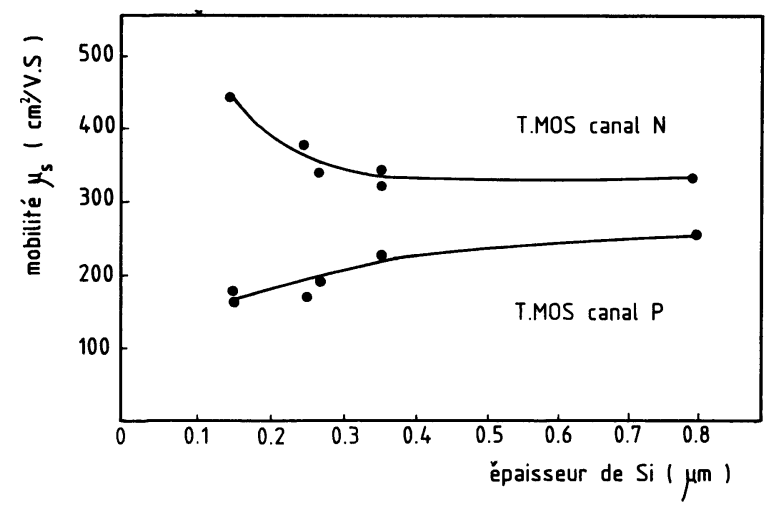

Fig. 3.12. - Mobilité en couche d'inversion pour un transistor MOS/SOS en fonction de l'épaisseur de Si épitaxié (Si (100)) (d'après Gris et Bello [42]).

[Inversion layer mobility for a MOS/SOS transistor versus epitaxied Si thickness (Si (100)) (after Gris and Bello [42]).] surface. En ce qui concerne le SOS, les densités de charges d'interface $Q_{\mathrm{ss}} \# 3 \times 10^{10}$ à $10^{11} \mathrm{~cm}^{-2}$, déduites du décalage des tensions de seuil [66], demeurent comparables aux valeurs mesurées à l'interface $\mathrm{SiO}_{2}$-Si massif. Cela justifie a posteriori que les valeurs de la mobilité en surface trouvées sur SOS soient proches de celles obtenues sur Si massif.

Englert et al. [70] ont étudié les oscillations de la magnétoconductance quantique sur des structures MOS/SOS sous champ magnétique intense. La masse cyclotron des électrons du canal $m_{\mathrm{c}}^{*}=0,5 m_{0}$ déduite de l'analyse de la structure des oscillations à basse température, est en accord avec la valeur théorique : $m_{\mathrm{c}}^{*}=0,42 m_{0}\left(m_{0}=\right.$ masse de l'électron $)$. Le facteur de dégénérescence $g_{\mathrm{v}}$ des vallées de la structure de bande à 2 dimensions (orientation (100)) trouvé égal à 2 , n'est pas compatible en revanche avec la théorie $\left(g_{v}=4\right)$. Cependant ce dernier point n'est pas une originalité du SOS car des valeurs analogues ont été trouvées sur des $\mathrm{MOS} / \mathrm{Si}$ massif, et demeurent à l'heure actuelle sans explication. Sur la figure 3.13, sont représentées à titre de comparaison les extrémas des oscillations Shubnikov-De Haas obtenues sur des TMOS réalisés sur silicium sur saphir et sur silicium sur spinel. Englert et al. [70] interprètent la différence observée comme une conséquence directe de l'aniso-
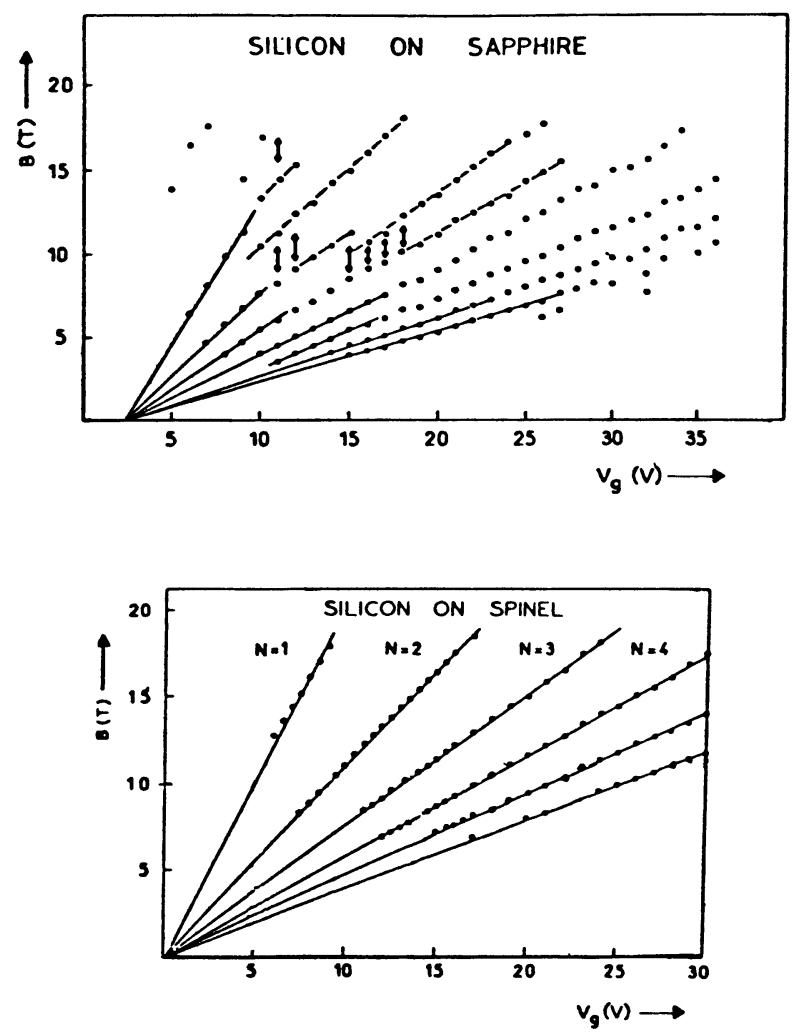

Fig. 3.13. - Extrema des oscillations Shubnikov-De Haas pour des MOSFET/SOS. Comparaison entre le $\mathrm{Si}$ sur saphir et le Si sur spinel (d'après Englert et al. [70]).

[Extrema of Shubnikov-De Haas oscillations for MOSFET/ SOS. Comparison between $\mathrm{Si}$ on sapphire and $\mathrm{Si}$ on spinel (after Englert et al. [70]).] 
tropie des contraintes film-substrat, engendrée dans le SOS par l'anisotropie de dilatation thermique du saphir (rhomboédrique), ce qui n'est pas le cas du spinel (cubique).

\subsection{Interface $\mathrm{Si}-\mathrm{Al}_{2} \mathrm{O}_{3}$.}

a) Etude indirecte. - Gentil [61] a montré expérimentalement l'existence sur les TMOS/SOS à canal $N$, d'un courant de fuite direct entre le drain et la source. Ce courant, qui varie exponentiellement avec la tension de polarisation du volume, révèle la présence d'un canal parasite à l'interface $\mathrm{Si}-\mathrm{Al}_{2} \mathrm{O}_{3}$ travaillant en régime d'inversion faible, dont on déduit une densité d'états à l'interface $\mathrm{Si}-\mathrm{Al}_{2} \mathrm{O}_{3}: N_{\mathrm{ss}} \# 10^{12} \mathrm{~cm}^{-2} \mathrm{eV}^{-1}$.

Gentil et Chausse [72], Choujaa [73] et Touboul et al. [74] ont mesuré la densité spectrale de bruit sur des TMOS/SOS en fonction de la polarisation du volume. Leurs résultats montrent que l'intensité du bruit augmente notablement lorsque la zone de charge d'espace du volume balaye la totalité de l'épaisseur de la couche de Si (Fig. 3.14a). La comparaison avec les mesures réalisées sur $\mathrm{Si}$ massif est éloquente et permet de conclure que l'origine du bruit excédentaire dans le cas du SOS est principalement due à une forte densité de centres de génération-recombinaison (G-R) croissante vers l'interface $\mathrm{Si}-\mathrm{Al}_{2} \mathrm{O}_{3}$. Compte tenu du dopage du substrat (\#10 $10^{15} \mathrm{~cm}^{-3}$ ), la tension de seuil à laquelle apparaît l'accroissement du bruit ne devrait excéder, théoriquement, 200 à $300 \mathrm{mV}$, alors qu'elle vaut expérimentalement 1 à $3 \mathrm{~V}$. On en conclut qu'il existe une forte charge fixe $Q_{\mathrm{sa}}$ localisée dans le saphir et estimée à $5 \times 10^{11} \mathrm{~cm}^{-2}$ [72]. L'analyse en fonction de la fréquence de la densité spectrale de bruit est caractéristique $\mathrm{du}$ bruit $\mathrm{G}-\mathrm{R}$ à plusieurs centres. Sur la figure $3.14 b$ on distingue deux fréquences de coupure correspondant à deux centres G-R distincts. De l'intensité du bruit du plateau extrapolé à basse fréquence, on déduit que la concentration de centres G-R ramenée à l'interface $\mathrm{Si}-\mathrm{Al}_{2} \mathrm{O}_{3}$ est comprise entre $4 \times 10^{11}$ et $2 \times 10^{12} \mathrm{~cm}^{-2}$ [72-74]. De plus, Gentil et Chausse [72] estiment que l'épaisseur sur laquelle sont répartis les centres $\mathrm{G}-\mathrm{R}$ est de l'ordre de $500 \AA$ à l'interface $\mathrm{Si}-\mathrm{Al}_{2} \mathrm{O}_{3}$, valeur confirmant celle trouvée par d'autres méthodes (cf. § 3.5).

b) Etude directe. - Choujaa [73] a étudié la capacité métal- $\mathrm{Al}_{2} \mathrm{O}_{3}-\mathrm{Si}$ par la méthode différentielle $C(V)$ à haute tension proposée originellement par Goodman [75]. L'interprétation des caractéristiques $C(V)$ à l'aide du modèle classique de Nicollian et Goetzberger [76] et du modèle cellulaire de Castagné [77] et Rossel [78] permet de donner les principaux paramètres de l'interface. Choujaa [73] met en évidence un surdopage de type $N$ sur $500 \AA$ au voisinage de l'interface $\mathrm{Si}-\mathrm{Al}_{2} \mathrm{O}_{3}$, tant sur des substrats de type $\mathrm{N}$ que de type $P$. En outre, il trouve une densité de charge fixe à l'interface $Q_{\mathrm{ss}} \# 2 \times 10^{11}$ à $10^{12} \mathrm{~cm}^{-2}$, qui confirme les valeurs obtenues indirectement. Enfin il précise,
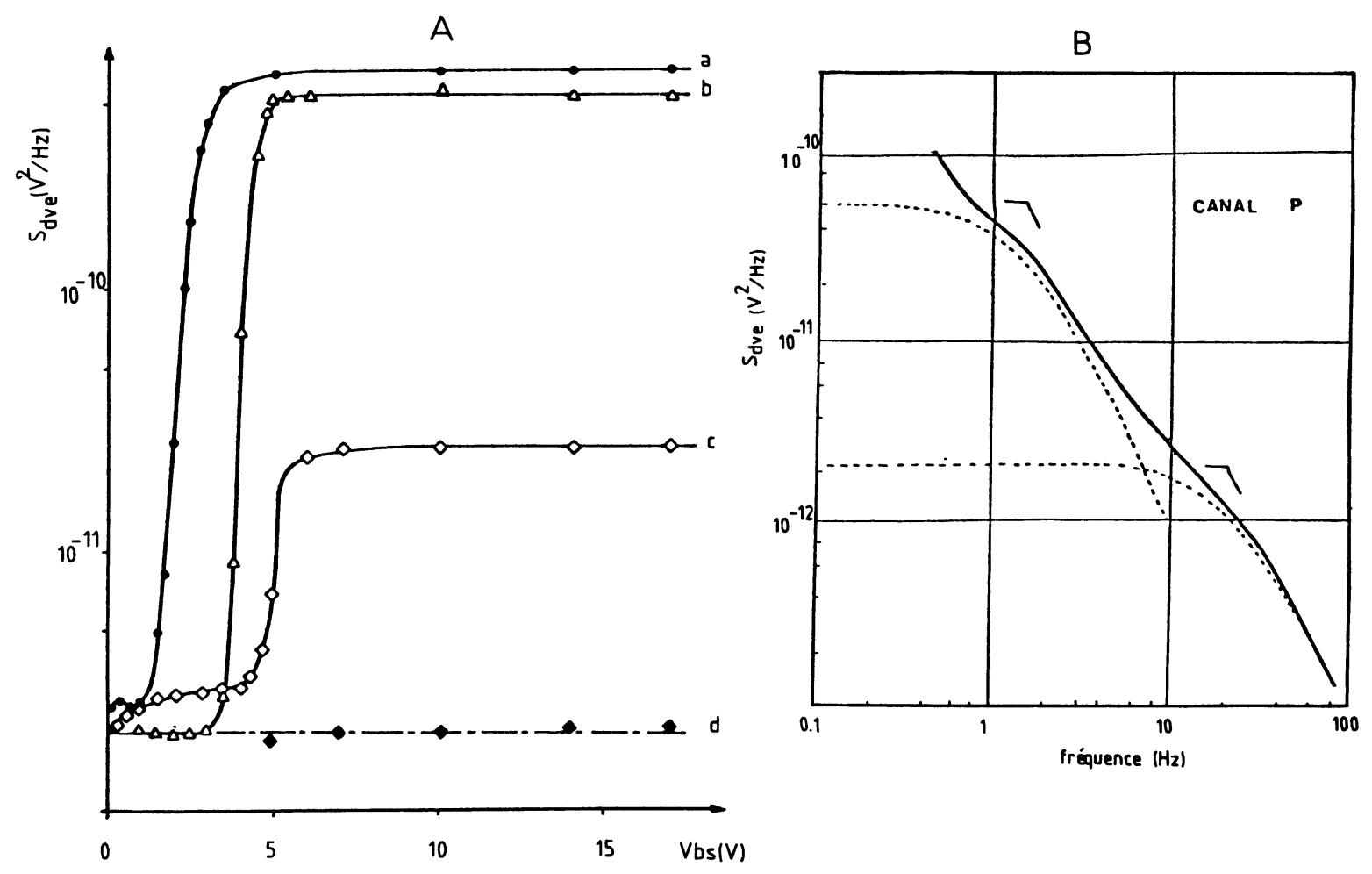

Fig. 3.14. - Densités spectrales de bruit en fonction : $a$ ) de la polarisation du volume obtenu sur des MOS/SOS (a, b, c) et sur un MOS sur Si massif (d) (fréquence $1 \mathrm{kHz}$ ) (d'après Gentil et Chausse [72]), b) de la fréquence (d'après Choujaa [73]).

[Noise spectral density as a function of : a) bulk bias obtained on MOS/SOS (a, b, c) and on MOS/bulk-Si (d) (frequency $1 \mathrm{kHz}$ ) (after Gentil and Chausse [72]), b) frequency (after Choujaa [73]).] 
grâce à une analyse fine des courbes $C(V)$, le profil de densité d'états $N_{\mathrm{ss}}$ à l'interface $\mathrm{Si}_{-} \mathrm{Al}_{2} \mathrm{O}_{3}$ (Fig. 3.15). La densité moyenne d'états de surface est donc plus importante qu'à l'interface $\mathrm{Si}_{-} \mathrm{SiO}_{2}$ et vaut typiquement quelque $10^{12} \mathrm{~cm}^{-2} \mathrm{eV}^{-1}$. Le maximum de la distribution énergétique indique la présence d'un niveau profond situé à $255 \mathrm{meV}$ de la bande de conduction, imputable aux défauts et contaminations dans la zone de transition à l'interface $\mathrm{Si}_{-} \mathrm{Al}_{2} \mathrm{O}_{3}$ [73]. Ces résultats sont en accord avec ceux de Goodman [75] mais n'ont pas été toutefois complètement confirmés par Dube et al. [79] qui n'observent pas de maximum au milieu du gap.

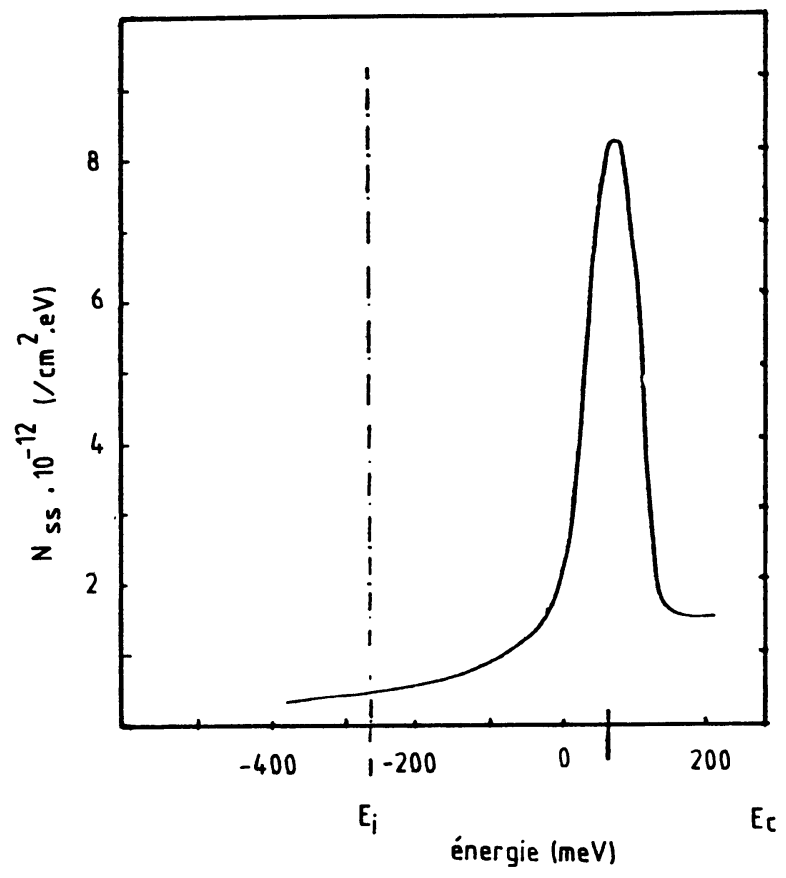

Fig. 3.15. - Profil de la densité d'états de surface à l'interface $\mathrm{Si}-\mathrm{Al}_{2} \mathrm{O}_{3}$ (d'après Choujaa [73]).

[Surface states density profile at the $\mathrm{Si}-\mathrm{Al}_{2} \mathrm{O}_{3}$ interface (after Choujaa [73]).]

Gris et Montier [65] ont étudié les caractéristiques $I_{\mathrm{d}}\left(V_{\mathrm{g}}\right)$ et $I_{\mathrm{d}}\left(V_{\mathrm{d}}\right)$ de transistors à déplétion $\mathrm{N}^{+} \mathrm{N}^{-} \mathrm{N}^{+}$ par effet de champ face arrière. Ils en déduisent une tension de bande plate à l'interface $\mathrm{Si}-\mathrm{Al}_{2} \mathrm{O}_{3}$ de l'ordre de 2 à $3 \mathrm{kV}$ pour une épaisseur de saphir de $250 \mu \mathrm{m}$, ce qui correspond à une densité de charges d'interface de l'ordre de $5 \times 10^{11} \mathrm{~cm}^{-2}$. La pente de la courbe $I_{\mathrm{d}}\left(V_{\mathrm{g}}\right)$ en régime de forte accumulation correspond à une mobilité de porteurs à l'interface $\mathrm{Si}_{-} \mathrm{Al}_{2} \mathrm{O}_{3}$ de l'ordre de 2 à $10 \mathrm{~cm}^{2} / \mathrm{Vs}$, valeur cent fois plus faible qu'à l'interface supérieure $\mathrm{Si}_{-} \mathrm{SiO}_{2}$.

Gentil [80] et Moreau [81] ont étudié le transistor métal- $\mathrm{Al}_{2} \mathrm{O}_{3}-\mathrm{Si}$ en régime d'inversion, après amincissement par abrasion du substrat de saphir à 30-40 $\mu \mathrm{m}$. La mobilité dans le canal $\mathrm{N}$ obtenue de la pente des caractéristiques $I_{\mathrm{d}}\left(V_{\mathrm{g}}\right)$ en forte inversion (Fig. 3.16), est de l'ordre de 10 à $40 \mathrm{~cm}^{2} / \mathrm{Vs}$. La densité de charges fixes présentes dans le saphir $Q_{\mathrm{sa}}$ est environ

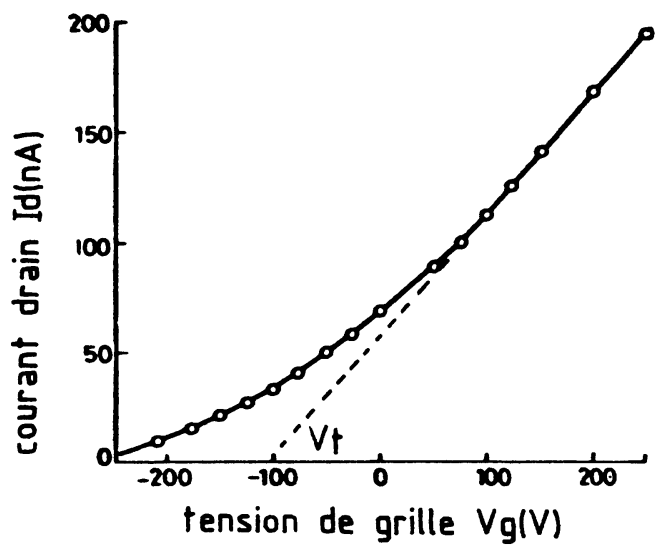

Fig. 3.16. - Caractéristique courant drain $I_{\mathrm{d}}$-tension de grille $V_{\mathrm{g}}$ face arrière, pour un TMOS/SOS de dimension $100 \times 100 \mu \mathrm{m}^{2}$ et d'épaisseur de saphir $80 \mu \mathrm{m}$ (d'après Gentil [80]).

[Drain current $I_{\mathrm{d}}$-back gate voltage $V_{\mathrm{g}}$ characteristic for TMOS/SOS of dimension $100 \times 100 \mu \mathrm{m}^{2}$ and sapphire thickness $80 \mu \mathrm{m}$ (after Gentil [80]).]

de $10^{12} \mathrm{~cm}^{-2}$. Enfin l'analyse des caractéristiques $I_{\mathrm{d}}\left(V_{\mathrm{g}}\right)$ en inversion faible permet de préciser la densité moyenne d'états d'interface

$$
N_{\mathrm{ss}} \# 1 \text { à } 7 \times 10^{12} \mathrm{~cm}^{-2} \mathrm{eV}^{-1}
$$

L'ensemble de ces résultats relatifs à l'interface Si$\mathrm{Al}_{2} \mathrm{O}_{3}$ ont été récemment confirmés et complétés par Brini et Gentil [82].

3.5 PropriÉTÉS DE RECOMBINAISON. - Les propriétés de recombinaison dans le SOS ont été essentiellement étudiées à l'aide de la photo-excitation et des caractéristiques $I(V)$ de dispositifs travaillant en obscurité.

3.5.1 Photoréponses transitoires. - Roux et BielleDaspet [41] ont analysé pour le cas du SOS de type $P$ (résistivité 0,7 à $2 \Omega \mathrm{cm}$, épaisseur du film 0,9 à $2 \mu \mathrm{m}$ ) les réponses transitoires de la photoconductivité et des photocourants à une excitation par laser de durée $30 \mathrm{~ns}$. La décroissance des photocourants de diffusion des porteurs majoritaires ou minoritaires présente deux composantes :

- une composante rapide qui suit la forme de l'impulsion laser et qui permet de conclure que la durée de vie effective dans le SOS est inférieure à $10 \mathrm{~ns}$;

- une composante lente qui fait apparaître une augmentation du nombre "effectif " de trous par rapport à l'équilibre (abaissement du niveau de Fermi) et ceci bien après que les porteurs photoinjectés se soient recombinés. Cette observation suggère l'existence d'amas de défauts qui peuvent capturer les minoritaires et qui se comportent comme de véritables régions isolantes : elles sont entourées d'une barrière de potentiel (dont l'effet est de diminuer la mobilité et la concentration effectives des trous), 
s'effaçant progressivement lors du piégeage des électrons photo-injectés.

Les zones isolantes permettent également d'expliquer la variation de la photoconductivité. Initialement $\sigma(t)$ augmente pendant un temps relativement long (2-3 $\mu$ s selon l'échantillon) ce qui correspond au remplissage des pièges. La décroissance de $\sigma(t)$ n'est pas, comme pour le matériau homogène ( $\mathrm{Si}$ massif), une simple exponentielle de laquelle on déduit la durée de vie. Les deux constantes de temps $\tau_{1,2}$ qui caractérisent le début et la fin de la décroissance de $\sigma(t)$ sont très longues; elles augmentent davantage lorsque la température est abaissée et se stabilisent ensuite vers $200 \mathrm{~K}$ (Fig. 3.17), comportement caractéristique de la réémission des électrons piégés. On en déduit le niveau énergétique des zones isolantes $E_{\mathrm{i}}=E_{\mathrm{c}}$ $0,17 \mathrm{eV}$ ainsi que leur niveau de Fermi interne : $E_{\mathrm{F}}=E_{\mathrm{v}}+0,32 \mathrm{eV}$. Ces zones sont responsables d'une réduction de l'ordre de $33 \%$ de la conductivité globale du P-SOS [41].

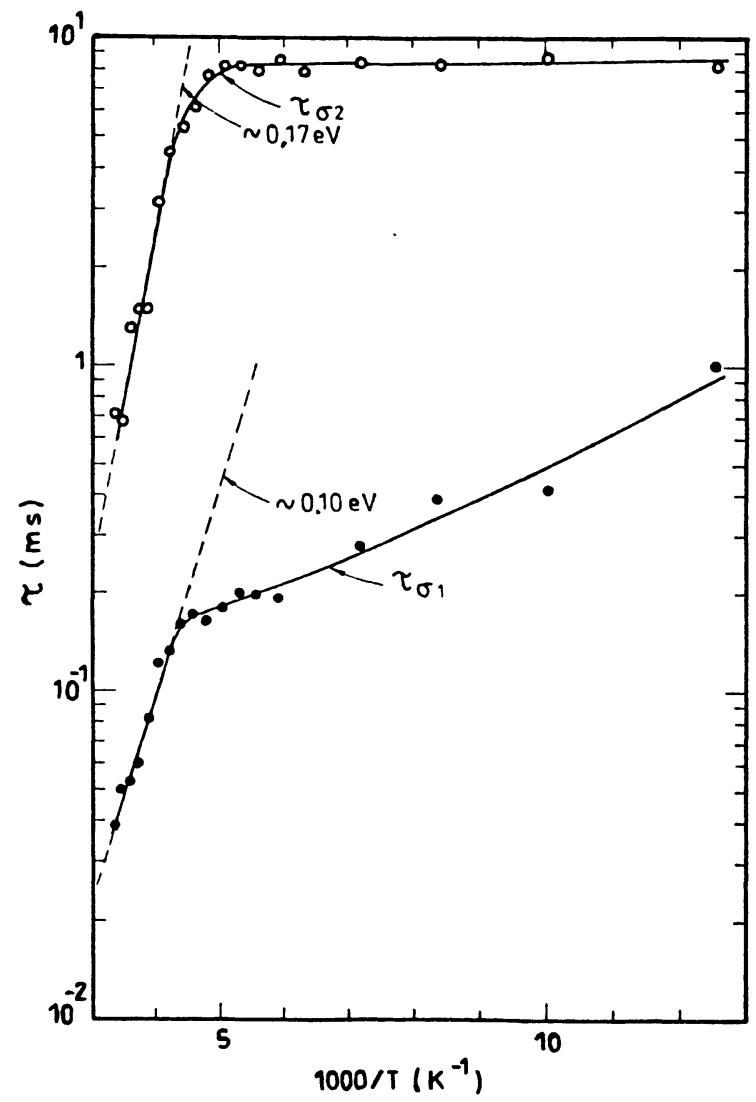

Fig. 3.17. - Influence de la température sur les constantes de temps $\tau_{1,2}$ de la décroissance de la photoconductivité. Echantillon SOS d'épaisseur $0,9 \mu \mathrm{m}$ et de dopage $N_{\mathrm{A}}=5 \times$ $10^{16} \mathrm{~cm}^{-3}$, soumis à une impulsion laser $(E=0,5 \mathrm{~mJ}$, $\lambda=1,06 \mu \mathrm{m}$ ) (d'après Roux et Bielle-Daspet [41]).

[Influence of temperature on the time constants $\tau_{1,2}$ of the photoconductivity decay for SOS samples of thickness $0.9 \mu \mathrm{m}$ and doping $5 \times 10^{16} \mathrm{~cm}^{-3}$ (laser pulse $E=0.5 \mathrm{~mJ}$, $\lambda=1.06 \mu \mathrm{m})($ after Roux et Bielle-Daspet [41]).]
Ces observations sont en bon accord avec les mesures du coefficient d'absorption du SOS qui s'avère être plus élevé que dans le Si massif pour des énergies des photons inférieures au gap. Dumin [83] met en évidence l'existence de précipités de défauts, situés à environ $0,3 \mathrm{eV}$ du bord de bande du SOS de type $\mathbf{N}$ ou $\mathbf{P}$. La densité de ces défauts augmente lorsque l'épaisseur de la couche diminue, ce qui relie naturellement la position des régions isolantes dans le film à son inhomogénéité (\$ 3.3 ). Plus récemment Lagowski et al. [74] expliquent les résultats de mesures de photovoltage par une amorphisation partielle du SOS (se traduisant par un gap $E_{\mathrm{g}}=1,45 \mathrm{eV}$ au lieu de $1,1 \mathrm{eV})$ dont le degré dépend, tout comme la densité de centres profonds, des traitements thermiques subis par le matériau.

3.5.2 Recombinaison en surface et profil de la durée de vie. - Tout particulièrement dans les couches minces, les processus de recombinaisons en volume et sur les surfaces sont intimement liés, de sorte que les mesures expérimentales ne fournissent qu'une valeur effective $\tau_{\text {eff }}$ de la durée de vie. Suivant le phénomène exploité et selon l'épaisseur des couches étudiées, $\tau_{\text {eff }}$ s'écarte plus ou moins de la durée de vie volumique $\tau_{\mathrm{v}}$, ce qui explique l'éventail des résultats publiés sur le SOS. L'étude des caractéristiques $I(V)$ directes des diodes diffusées [85] et l'analyse transitoire de la décroissance de conductivité après irradiation par électrons d'énergie 4 à $20 \mathrm{keV}$ [86] conduisent à une durée de vie de l'ordre de $10^{-10} \mathrm{~s}$, alors que des résultats de microscopie électronique [87] et de courants de fuite des capacités MOS [88] suggèrent des valeurs 100 fois plus élevées. De plus, il semble que le fort dopage de l'interface $\mathrm{Si}_{-} \mathrm{Al}_{2} \mathrm{O}_{3}$, au phosphore $\left(N_{\mathrm{D}}=10^{20} \mathrm{~cm}^{-3}[89]\right)$ ou à l'arsenic $\left(N_{\mathrm{D}}>5 \times 10^{16} \mathrm{~cm}^{-3}\right.$ [88]) entraîne, par un effet de "gettering", l'augmentation de $\tau_{\mathrm{v}}$ d'au moins un ordre de grandeur; une valeur de $\tau_{\mathrm{v}}=2 \mu \mathrm{s}$ a même été rapportée [89].

Senn [90] a analysé les caractéristiques des transistors bipolaires latéraux et des diodes sur SOS; en utilisant l'expression $\tau_{\text {eff }}^{-1}=\tau_{\mathrm{v}}^{-1}+s_{1} / b$ et en supposant $\tau_{\mathrm{v}}=50 \mathrm{~ns}$ il détermine une vitesse de recombinaison $s_{1}$ à l'interface $\mathrm{Si}_{-} \mathrm{Al}_{2} \mathrm{O}_{3}$ de l'ordre de $10^{3} \mathrm{~m} / \mathrm{s}$. Ce résultat paraît en accord avec celui de Garcia [68] qui attribue l'essentiel du courant de fuite $I_{\mathrm{s}}$ des diodes $\mathrm{P}^{+} \mathrm{NN}^{+}$à grille de contrôle aux processus de recombinaison-génération à l'interface $\mathrm{Si}_{-} \mathrm{Al}_{2} \mathrm{O}_{3}$ $\left(I_{\mathrm{s}} \propto s_{1}\right)$. Par ailleurs, la mesure de la résistance série de la base $\mathbf{N}$ des diodes lui a permis d'évaluer, à travers un modèle très simplifié, la longueur « effective " de diffusion des minoritaires : $L_{\mathrm{p}}=0,7 \mu \mathrm{m}$ à faible injection et $L_{\mathrm{p}}=7 \mu \mathrm{m}$ à forte injection. Une confirmation de la valeur minimale $(0,7 \mu \mathrm{m})$ est apportée par l'évolution du courant induit dans les diodes $\mathrm{P}^{+} \mathbf{N}$ lorsqu'un faisceau d'électrons (provenant d'un microscope électronique à balayage) se rapproche de la jonction; de plus, Garcia [68] montre 


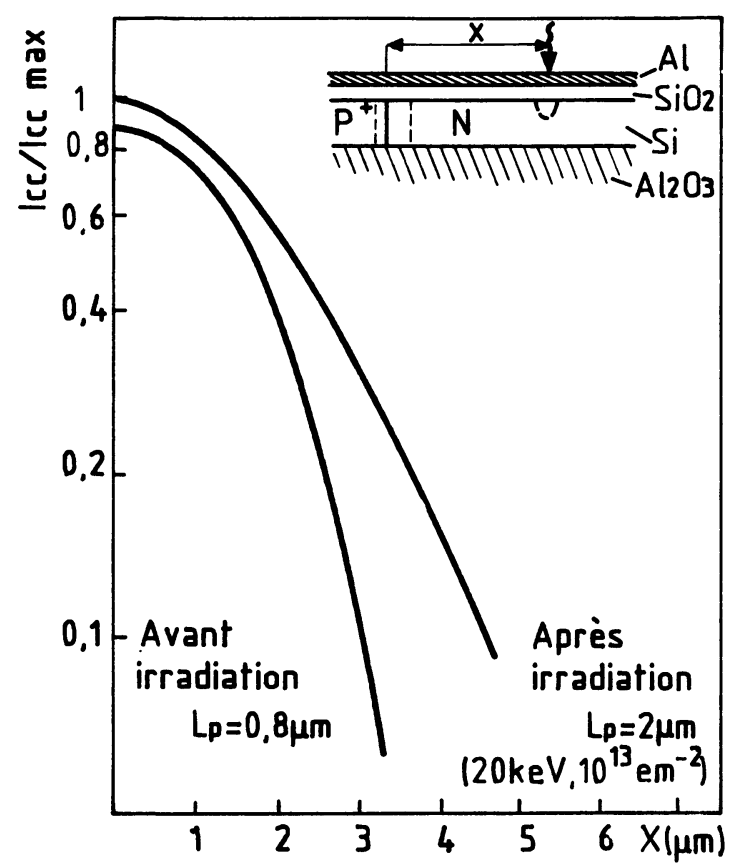

Fig. 3.18. - Enregistrement du courant induit lors de la mesure de $L_{\mathrm{p}}$ par MEB (d'après Garcia [68]).

[Injected current plot during the $L_{\mathrm{p}}$ measurements by scanning electron microscopy (after Garcia [68]).]

(Fig. 3.18) qu'après irradiation des échantillons à $20 \mathrm{keV}$ et $10^{13}$ électrons $/ \mathrm{cm}^{2}, L_{\mathrm{p}}$ augmente jusqu’à $2 \mu \mathrm{m}$, ce qui tend à prouver l'écrantage partiel de l'interface $\mathrm{Si}_{-} \mathrm{Al}_{2} \mathrm{O}_{3}$.

Les études entreprises par Choujaa [73] concernent en particulier :

- le courant de fuite des transistors MOS et des diodes PN (drain-substrat et source-substrat),

- le courant transitoire des capacités MOS lorsqu'une impulsion fait passer d'un régime d'accumulation ou d'inversion à un régime de désertion profonde,

- le courant inverse des diodes $\mathrm{P}^{+} \mathrm{NN}^{+}$et $\mathrm{P}^{+} \mathrm{PN}^{+}$, contrôlé par deux grilles situées sur les faces avant $\mathrm{Al}-\mathrm{SiO}_{2}-\mathrm{Si}$ et arrière $\mathrm{Al}_{-}-\mathrm{Al}_{2} \mathrm{O}_{3}-\mathrm{Si}$ (Fig. 3.19).

De manière générale le courant de fuite augmente pendant l'extension de la désertion et se sature (tout comme le bruit [72, 73], cf. § 3.4) lorsque la zone de charge d'espace s'étale jusqu'à l'interface $\mathrm{Si}-\mathrm{Al}_{2} \mathrm{O}_{3}$. Choujaa [73] déduit une durée de vie de 1 à $10 \mathrm{~ns}$ et une vitesse de recombinaison $\left(\mathrm{Si}-\mathrm{Al}_{2} \mathrm{O}_{3}\right)$ de l'ordre de $200 \mathrm{~m} / \mathrm{s}$.

Cette valeur est inférieure aux estimations précédentes mais reste très supérieure à celle correspondant à l'interface $\mathrm{Si}_{-} \mathrm{SiO}_{2}$; l'activation en température des courants de fuite montre que les centres de recombinaison sont situés à environ $0,3 \mathrm{eV}$ au-dessus du milieu de la bande interdite.

Les résultats expérimentaux de la figure 3.19 ont été complétés par Gris et Montier [65]. Ils démontrent que les courants inverses des diodes sur SOS

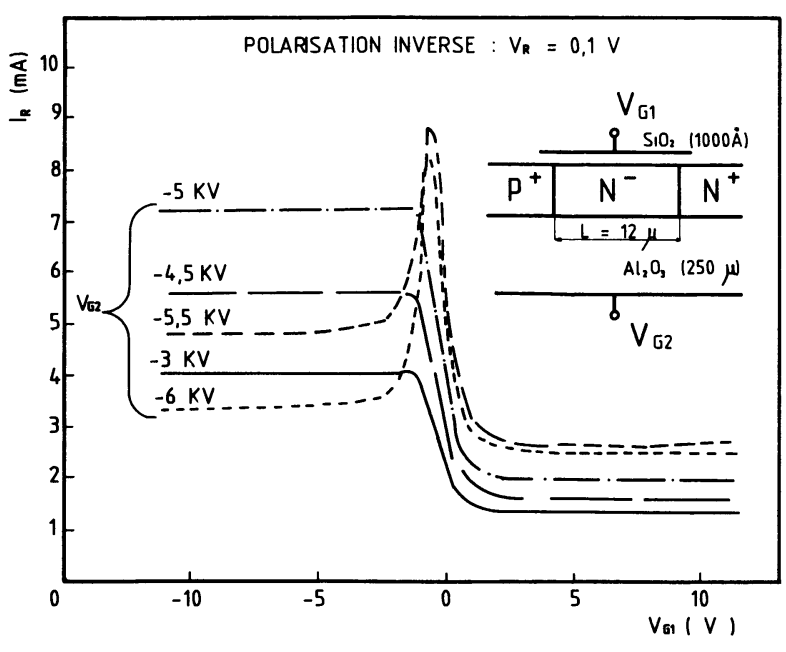

Fig. 3.19. - Courant inverse d'une diode $\mathrm{P}^{+} \mathrm{NN}^{+}$en fonction des tensions appliquées sur les deux grilles (d'après Choujaa [73]). Pour $V_{\mathbf{g}_{1}}>0$, lorsque $\left(-V_{\mathbf{g}_{2}}\right)$ augmente l'interface $\mathrm{Si}-\mathrm{Al}_{2} \mathrm{O}_{3}$ passe d'un régime d'accumulation à la désertion ( $I_{\mathrm{s}}$ augmente) et ensuite à l'inversion ( $I_{\mathrm{s}}$ se sature). Pour $V_{\mathbf{g}_{1}}<0, I_{\mathrm{s}}$ augmente avec $\left(-V_{\mathbf{g}_{2}}\right)$ pendant la désertion et diminue en régime d'inversion (écrantage de l'interface $\mathrm{Si}-\mathrm{Al}_{2} \mathrm{O}_{3}$ pour $V_{\mathrm{g}_{2}}<-5 \mathrm{kV}$ ). Le pic de $I_{\mathrm{s}}$ correspond à une désertion totale du $\mathrm{Si}$ (pas d'accumulation ou d'inversion aux interfaces [65]).

[Reverse current of $\mathrm{P}^{+} \mathrm{NN}^{+}$diode as a function of foreward and backward gate biasing (after Choujaa [73]).]

sont dus aux générations en volume, qui masquent donc l'effet de la génération à l'interface $\mathrm{Si}-\mathrm{Al}_{2} \mathrm{O}_{3}$.

Pour mieux dissocier recombinaison en volume et sur les surfaces Cristoloveanu et al. [91] et Lilienkamp et Pfleiderer [92] ont proposé l'utilisation de l'effet magnétodiode. Dans une diode $\mathrm{P}^{+} \mathrm{NN}^{+}$(Fig. 3.20) soumise à l'action d'un champ magnétique transversal, les électrons et les trous subissent une déviation Lorentzienne : pour $B<0$ ils sont poussés vers l'interface très recombinante $\mathrm{Si}-\mathrm{Al}_{2} \mathrm{O}_{3}$ de sorte que leur nombre moyen $\langle\delta n\rangle$ et le courant $I$ diminuent ; au contraire, pour $B>0$, ils sont soustraits à l'action de cette interface et alors $\langle\delta n\rangle$ et $I$ augmentent.

La théorie générale de l'effet magnétodiode $[37,93]$ montre que la caractéristique $I(V)$ du régime semiconducteur s'écrit :

$$
I=\frac{9}{8} e \mu_{\mathrm{n}} \mu_{\mathrm{p}} \tau_{\mathrm{eff}}\left(n_{0}-p_{0}\right) \frac{V^{2}}{L^{3}}
$$

où $L$ est la longueur de la base et $\tau_{\text {eff }}$ dépend de façon complexe des paramètres de recombinaison et de l'intensité des champs. En particulier $\tau_{\text {eff }}$ est très sensible à la variation de $B$ ce qui permet aux magnétodiodes d'être d'excellents capteurs magnétiques [94]. Dans une structure " épaisse » $(b=7 \mu \mathrm{m}$. Fig. 3.20b), la durée de vie volumique $\tau_{\mathrm{v}}$ peut être considérée comme quasi uniforme et 3 points expérimentaux $(+B,-B$ et $B=0)$ suffisent pour déterminer les valeurs moyennes de $\tau_{\mathrm{v}} \# 10$ ns et des deux 


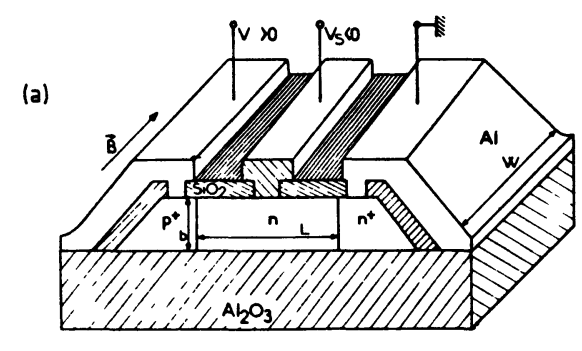

(c)
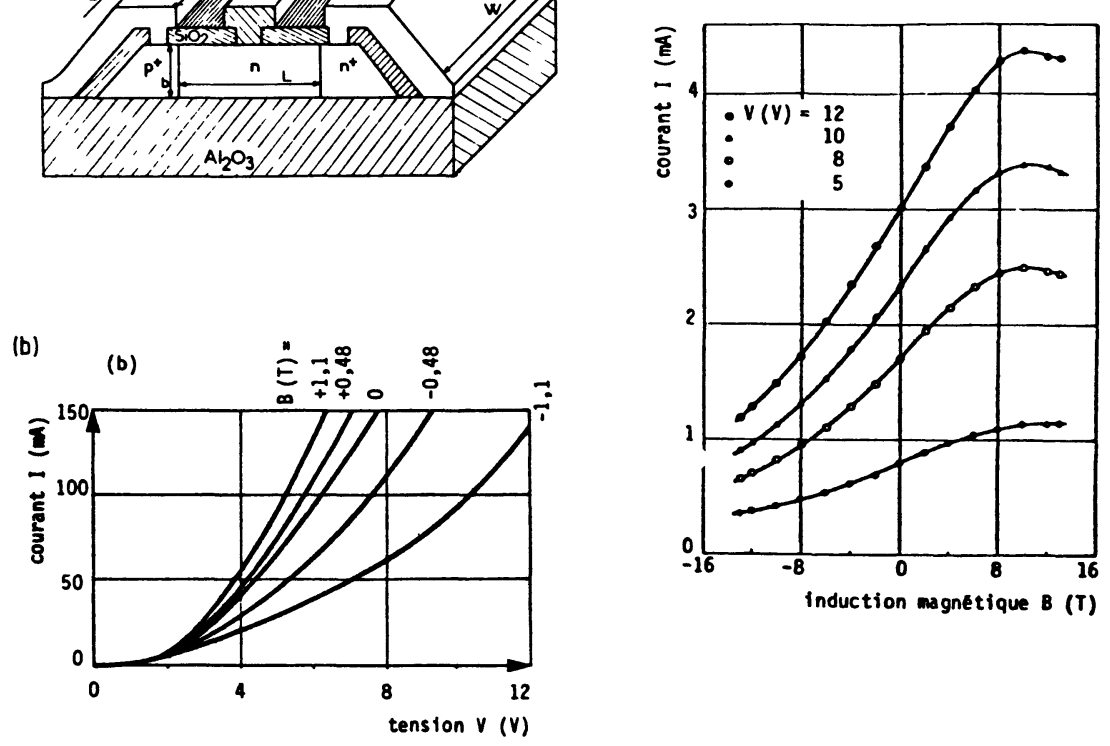

Fig. 3.20. - Configuration d'une magnétodiode SOS (Fig. $a$ ) et caractéristiques expérimentales à faible champ (Fig. $b$ : $L=18 \mu \mathrm{m}, W=200 \mu \mathrm{m}, n_{0}=10^{16} \mathrm{~cm}^{-3}, b=7 \mu \mathrm{m}$ ) et à fort champ magnétique (Fig. $c: L=10 \mu \mathrm{m}, W=50 \mu \mathrm{m}$, $b=0,65 \mu \mathrm{m}, n_{0}=2 \times 10^{16} \mathrm{~cm}^{-3}$ ) (d'après [37, 52, 91, 93, 94]).

[SOS magnetodiode configuration (Fig. $a$ ) and experimental characteristics at low field (Fig: $b: L=18 \mu \mathrm{m}, W=200 \mu \mathrm{m}$, $n_{0}=10^{16} \mathrm{~cm}^{-3}, b=7 \mu \mathrm{m}$ ) and at high magnetic field (Fig. $c: L=10 \mu \mathrm{m}, W=50 \mu \mathrm{m}, b=0.65 \mu \mathrm{m}, n_{0}=2 \times 10^{16} \mathrm{~cm}^{-3}$ ).]

vitesses de recombinaisons $s_{1}\left(\mathrm{Si}_{-}-\mathrm{Al}_{2} \mathrm{O}_{3}\right) \# 180 \mathrm{~m} / \mathrm{s}$ et $s_{2}\left(\mathrm{Si}_{-} \mathrm{SiO}_{2}\right) \# 15 \mathrm{~m} / \mathrm{s}[37,52]$.

Les films usuels d'épaisseur plus faible nécessitent de prendre en compte la réduction de la durée de vie vers l'interface $\mathrm{Si}-\mathrm{Al}_{2} \mathrm{O}_{3}$. La réponse transitoire des capacités MOS et l'évolution des courants de fuite, en régime de déplétion, des diodes à grille de contrôle suggèrent une distribution exponentielle des centres G-R [88, 95]. Par ailleurs, les analyses SIMS [24] et les mesures de bruit sur les transistors MOS à déplétion profonde [72] apportent des arguments sérieux en faveur d'une plus forte densité de centres dans les premières centaines d'angströms. On montre de plus que les caractéristiques des magnétodiodes à haut champ magnétique (Fig. 3.20c) [96], ne peuvent être retrouvées théoriquement ni pour une distribution uniforme de $\tau_{\mathrm{v}}$, ni lorsque la variation de $\tau_{\mathrm{v}}(y)$ excède un ordre de grandeur. Le tableau I résume les paramètres déduits pour les deux modèles d'inhomogénéité de $\tau_{\mathrm{v}}(y)$, en considérant des lois générales de recombinaison :

$$
\begin{aligned}
\tau_{\mathrm{v}}(y) & =\tau_{\mathrm{v}}^{0}(y) \frac{1+A_{\mathrm{v}} \delta n(y) /\left(n_{0}+p_{0}\right)}{1+\delta n(y) /\left(n_{0}+p_{0}\right)} \\
s_{1,2} & =s_{1,2}^{0} \frac{1+\delta n_{1,2} /\left(n_{0}+p_{0}\right)}{1+A_{s_{1,2}} \delta n_{1,2} /\left(n_{0}+p_{0}\right)} .
\end{aligned}
$$

Cette simulation confirme d'abord l'existence d'une durée de vie inhomogène de 1 à 10 ns et ensuite la
Tableau I. - Paramètres de recombinaison obtenus à l'aide de l'effet magnétodiode pour une distribution uniforme (couche SOS d'épaisseur $7 \mu \mathrm{m}$ ), exponentielle ou abrupte (épaisseur $0,65 \mu \mathrm{m})$ de la durée de vie et pour des lois de recombinaison linéaires ou non linéaires (d'après Cristoloveanu et al. [52]).

[Recombination parameters deduced from the magnetodiode effect by considering uniform (SOS layer $7 \mu \mathrm{m}$ thick), exponential or abrupt $(0,65 \mu \mathrm{m}$ thick) distributions of the bulk lifetime and linear or non linear

\begin{tabular}{|c|c|c|c|c|c|c|c|c|}
\hline Modète & $\begin{array}{l}T_{V}\left(S_{1}-\right. \\
\left.S_{102}\right)(n s)\end{array}$ & $\left|\begin{array}{l}\tau_{v}^{0}\left(S_{1}-\right. \\
A(203)(n s)\end{array}\right|$ & $A_{V}$ & $\begin{array}{c}\mathrm{s2}\left(\mathrm{S} 1-\mathrm{SiO}{ }_{2}\right. \\
\left(\mathrm{ms} \mathrm{s}^{-}\right)\end{array}$ & $A_{S_{2}}$ & $\begin{array}{l}\mathrm{si}(\mathrm{Si}- \\
\mathrm{Al} 2 \mathrm{O})\left(\mathrm{ms}^{-1}\right.\end{array}$ & $A_{S_{1}}$ & $t_{s_{1}}=s_{(\dot{A})}$ \\
\hline $\begin{array}{l}\tau_{V}^{0}(y) \text { uniforme } \\
\text { recomb. } \\
\text { linéaires }\end{array}$ & 10 & 10 & 1 & $<15$ & 1 & 180 & 1 & 18000 \\
\hline $\begin{array}{l}T^{0}(y) \text { exponent } \\
\text { recomb }\end{array}$ & 50 & 5 & 0,5 & $<5$ & 0,5 & $20 \div 40$ & 0.7 & 1000 \\
\hline $\begin{array}{l}T_{y}^{0}(y) \text { abrupt } \\
\text { recomb. } \\
\text { non-linéaires }\end{array}$ & 7 & 0.5 & 0.7 & $<10$ & 0,5 & $30 \div 40$ & 0.5 & 100 \\
\hline
\end{tabular}
recombination laws (after Cristoloveanu et al. [52])].

bonne qualité de l'interface $\mathrm{Si}_{-} \mathrm{SiO}_{2}$. Soulignons qu'à l'interface film-substrat, la vitesse de recombinaison n'est pas très élevée mais modérée. Ce résultat en excellent accord avec les travaux de Gris et Montier [65], est expliqué par la continuité du taux de recombinaison sur la surface et dans le volume sousjacent : la décroissance de $\tau_{\mathrm{v}}(y)$ signifie que le volume est en réalité responsable d'une partie des recombinaisons qui avaient été précédemment attribuées à l'interface 
$\mathrm{Si}-\mathrm{Al}_{2} \mathrm{O}_{3}[68,73,90]$. Pour comparer les taux respectifs de recombinaison volumique et surfacique, une « épaisseur caractéristique de la surface" a été définie $\left(t_{\mathrm{s}} \# s_{1} \tau_{\mathrm{v}}\right)$; la continuité est obtenue pour $t_{\mathrm{s}}=100$ à $1000 \AA$, résultat qui indique la profondeur de l'influence de l'interface $\mathrm{Si}-\mathrm{Al}_{2} \mathrm{O}_{3}$ dans le volume [52] et qui se compare favorablement aux mesures plus directes de la couche de transition $\left({ }^{3}\right)$.

\section{Discussion et conclusion.}

4. 1 COHÉRENCE DES RÉSULTATS. - La cohérence des résultats entre travaux d'origines différentes mais concernant le même thème a été systématiquement soulignée au cours des sections précédentes. L'objet de ce paragraphe est de discuter la cohésion d'ensemble des résultats obtenus sur le SOS compte tenu de l'éventail des moyens de caractérisation utilisés. A cette fin nous avons regroupé dans le tableau II les principales informations résultant des diverses investigations que nous avons passées en revue. Nous avons distingué les études faites sur du SOS « non processé " (c'est-à-dire n'ayant pas subi d'opérations technologiques, telles que oxydation, recuit, métallisation..., nécessaires à la fabrication d'un dispositif), de celles réalisées sur du SOS "processé " ou sur des composants SOS.

Pour chacune des méthodes utilisées nous rappelons (tableau II) les conditions expérimentales, les résultats significatifs ainsi que les limitations inhérentes. Ce tableau nous permet de dégager les éléments clefs qui gouvernent les propriétés du matériau SOS réalisé et étudié en France.

La couche de transition située à l'interface Sisubstrat, d'épaisseur de quelques centaines d'Angströms, a été révélée par spectroscopie électronique; mise par ailleurs en évidence grâce aux propriétés électriques, elle se manifeste par la dégénérescence du matériau à basse température. Les propriétés de transport et de recombinaison dans cette couche proche de l'interface $\mathrm{Si}-\mathrm{Al}_{2} \mathrm{O}_{3}$ sont caractéristiques d'une dégradation très nette de la qualité cristalline. Cela se traduit par une forte diminution de la mobilité (10 à $20 \mathrm{~cm}^{2} /$ Vs selon les auteurs), associée à une

$\left.{ }^{3}\right)$ Ces mêmes dispositifs $\mathrm{P}^{+} \mathrm{NN}^{+}$ont servi à l'étude de l'échauffement de porteurs dans le SOS et de son influence sur les phénomènes de double injection. Dufour et Cristoloveanu [97, 98] montrent que la mobilité des porteurs commence à dépendre du champ électrique au-dessus de $2,5 \mathrm{kV} / \mathrm{cm}$ (valeur proche de celle du Si massif [99] et suit une loi approximative $\mu=$ Cte. $E^{-0,5}$; en raison de la faible mobilité à l'interface $\mathrm{Si}-\mathrm{Al}_{2} \mathrm{O}_{3}$, la vitesse de saturation n'est globalement atteinte qu'à des champs largement supérieurs à ceux du Si massif. Ils montrent par une méthode de double régionalisation, que la loi quadratique du régime semiconducteur (Eq. 3.15) se transforme en présence de porteurs chauds en une loi linéaire [98].
Tableau II. - Récapitulation des résultats obtenus sur couches SOS non processées, semi-processées et/ou processées par des méthodes de caractérisations physicochimiques et électriques.

[Summary of the results obtained on non-processed, semi-processed and/or processed SOS films by physico-chemical and electrical characterization.]

\begin{tabular}{|c|c|c|c|c|c|}
\hline \multicolumn{2}{|c|}{ EXPERIENCES } & \begin{tabular}{|l|} 
CONDIIIONS \\
EXPERIMENTALES \\
\end{tabular} & $\begin{array}{l}\text { RESULTATS } \\
\text { PRINCIPAUX }\end{array}$ & \begin{tabular}{|c|} 
RESULTATS \\
COMPLEMENTAIRES \\
\end{tabular} & $\begin{array}{c}\text { EXIGENCES } \\
\text { ET/OU } \\
\text { RESTRICTIONS }\end{array}$ \\
\hline \multirow{5}{*}{ 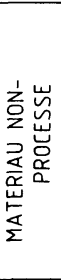 } & $\begin{array}{c}T \\
1\end{array}$ & TRANSMISSION & \begin{tabular}{c|} 
MUCLEATION \\
DISTORSION RESEAU
\end{tabular} & CONTRAINTES & $\begin{array}{c}\text { COUCHES NON- } \\
\text { COALESCEES }\end{array}$ \\
\hline & SIMS & - & PROFLL IMPURETES & $\begin{array}{l}\text { DOSE } \\
\text { COUCHE TRANITION }\end{array}$ & DESTRUCTIF \\
\hline & $R X$ & 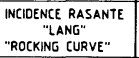 & $\begin{array}{l}\text { RUGOSITE } \mathrm{S}_{1} / \mathrm{Al}_{2} \mathrm{O}_{3} \\
\text { MOSAICTIE }\end{array}$ & \begin{tabular}{l|} 
STOECHOMETRIE \\
ISURFACE S1/AI/ \\
CONTRAINTES \\
CONTES
\end{tabular} & 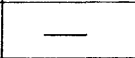 \\
\hline & RAMAN & - & CONTRAINTES & \begin{tabular}{|l|} 
CONFIIUURATION \\
OES BANOES \\
( "SPLITTING" ) \\
\end{tabular} & - \\
\hline & aUGer & - & $\begin{array}{c}\text { TAUX DE } \\
\text { CARBONE } \\
\end{array}$ & $\begin{array}{c}\text { PROFI } \\
\text { IMPURETTES }\end{array}$ & $\begin{array}{c}\text { DESTRUCTIF SI } \\
\text { ABRASION }\end{array}$ \\
\hline \multirow{4}{*}{ 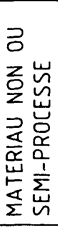 } & RESISTIVITE & \begin{tabular}{|c|} 
T ET ECLAAREMENT \\
VARIABLES
\end{tabular} & $\begin{array}{l}\text { OEGENERESCENCE } \\
\text { FLUCTUATION } \\
\text { POTENTIEL }\end{array}$ & 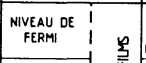 & 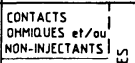 \\
\hline & \multirow{3}{*}{\begin{tabular}{lll} 
& HALL \\
\multirow{2}{n}{} & PTE \\
& \\
& MAGNETORESISTANCE \\
\end{tabular}} & T ET B VARIABLES & $\begin{array}{l}\text { MOBBLITE } \\
\text { ENEREIID DONSATION } \\
\text { COUCHE DEGENEREE }\end{array}$ & \begin{tabular}{|l|l|} 
FLUTT POT & $\overline{\bar{u}}$ \\
ANISOTROPIE & $\bar{L}$ \\
\end{tabular} & 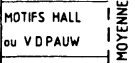 \\
\hline & & T VARIABLE & $\begin{array}{l}\text { COUCHE DEGENEREE } \\
\text { NIVEAU DE FERMI: }\end{array}$ & MOBUITE I & \begin{tabular}{|c|}
$\begin{array}{c}\text { MOTIFS } \\
\text { LONGS }\end{array}$ \\
\end{tabular} \\
\hline & & $\begin{array}{l}\text { LONGITUDINALE ET } \\
\text { TRANSVERSALE }\end{array}$ & $\begin{array}{l}\text { COEF ANISOTROPIE } \\
\text { CI }=77 \text {. } \\
\text { MOOE COLLISION }\end{array}$ & 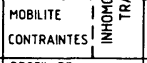 & 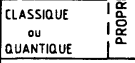 \\
\hline \multirow{6}{*}{ 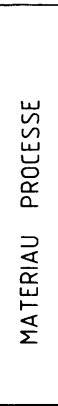 } & MOS - HALL & T ET \& VARIABLES & $\begin{array}{l}\text { PROFID DE } \\
\text { - CONOUCTIVITE } \\
\text { - MOBLITE } \\
\text {-CONCENTRATION } \\
\end{array}$ & $\begin{array}{l}\text { PROFIL DE } \\
\text {-MOEE DE COLLISION } \\
\text {-ANISOTROPIE }\end{array}$ & $\begin{array}{l}\text { OEPLETION } \\
\text { PROFONDE }\end{array}$ \\
\hline & capacite mos & $\begin{array}{l}\text { FACE AV ET AR } \\
\text { T VARIABLE }\end{array}$ & $\begin{array}{l}\text { PROFIL DOPAGE } \\
\text { CHARGES INTERFACES } \\
\text { DITRRBBUTTON ETATS } \\
\text { DINTERFACE } \\
\end{array}$ & $\begin{array}{l}\text { IMPURETES NON - } \\
\text { INTENTIONNELLES } \\
\text { FUUCT POTENTIEL } \\
\text { MOBILTE }\end{array}$ & 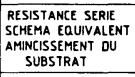 \\
\hline & $\begin{array}{l}\text { IRANSISTOR MOS } \\
\end{array}$ & $\begin{array}{l}\text { FACE AV ET AR } \\
\text { I ET B VARIABLES }\end{array}$ & 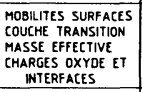 & \begin{tabular}{|l|} 
CONTRANTES \\
FACTEUR DE \\
DEGENERESCENCE \\
\end{tabular} & $\begin{array}{l}\text { MODELE TMOS } \\
\text { HAUT B } \\
\text { ( SHUBNIKOV । }\end{array}$ \\
\hline & IMAGNETODIODE & B VARIABLE & 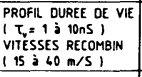 & $\begin{array}{l}\text { COUCHE TRANSITION } \\
\text { MOBIITE } \\
\text { PORTEURS CHAUDS }\end{array}$ & $\begin{array}{l}\text { LONGUEUR, T ET } \\
\text { DOPAEE POUR } \\
\text { DOUBLE-INJECTION } \\
\end{array}$ \\
\hline & I PHOTO-COURANTS & $\begin{array}{l}\text { LASER IMPULSION } \\
\text { T VARIABLE } \\
\end{array}$ & $\begin{array}{l}\text { 2ONES ISOLANTES } \\
\text { PIEGE VOLLUMES } \\
\left(1, E_{1}, E_{f}\right)\end{array}$ & $\begin{array}{c}\text { DUREE DE VIE } \\
\left(T_{v}<40 \text { NS }\right)\end{array}$ & ROLE DES SURFACES \\
\hline & 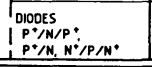 & $\begin{array}{l}\text { EFFETS DE CHAMP } \\
\text { AV ET AR } \\
\end{array}$ & $\begin{array}{l}\text { OUREE DE VIE } \\
\tau_{v}=1 \text { : } 10 \text { nS } \\
\end{array}$ & 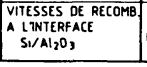 & $\begin{array}{l}\text { CONTROLE DE LA } \\
\text { CHARGE OESPACE }\end{array}$ \\
\hline
\end{tabular}

densité élevée de pièges $\left(1 \mathrm{à} 7 \times 10^{12} \mathrm{~cm}^{-2}\right)$ et de charges de surfaces $\left(10^{12} \mathrm{~cm}^{-2}\right)$. Bien que les phénomènes de recombinaison soient principalement dominés par le volume $\left(\tau_{\mathrm{v}}=1\right.$ à $\left.10 \mathrm{~ns}\right)$ c'est toutefois la qualité médiocre de l'interface $\mathrm{Si}-\mathrm{Al}_{2} \mathrm{O}_{3}$ qui provoque une recombinaison intense dans la couche de transition.

La non-uniformité des couches et leur caractère inhomogène, analysés par spectroscopie d'ions secondaires et par spectroscopie Auger sont reliés à l'existence d'un profil de défauts et d'impuretés non intentionnelles croissant vers l'interface $\mathrm{Si}-\mathrm{Al}_{2} \mathrm{O}_{3}$ et provenant de la contamination du substrat en cours d'épitaxie. La détermination directe des inhomogénéités de transport nécessite la combinaison de plusieurs types de mesures magnétoélectriques (conductivité, MR longitudinale et transversale, effet Hall, capacité MOS, ...), effectuées dans de larges gammes de température et d'induction magnétique et contrôlées par effet de champs. De manière générale ces mesures ont permis de corréler sans ambiguitté la détérioration des qualités de transport vers l'interface $\mathrm{Si}-\mathrm{Al}_{2} \mathrm{O}_{3}$, avec l'augmentation de la densité d'impuretés et de défauts.

La mobilité de dérive des électrons (déduite des 
courbes de MR longitudinale) est $50 \%$ supérieure à la mobilité de Hall et diminue d'un facteur 2 sur environ $0,4 \mu \mathrm{m}$ en dessous de l'interface $\mathrm{Si}_{-} \mathrm{SiO}_{2}$ où $\mu_{\mathrm{n}} \# 1000 \mathrm{~cm}^{2} / \mathrm{Vs}$; les mobilités dans le canal des TMOS sont typiquement $\mu_{\mathrm{n}} \# 500 \mathrm{~cm}^{2} / \mathrm{Vs}$ et $\mu_{\mathrm{p}} \# 200 \mathrm{~cm}^{2} /$ Vs. Par rapport au Si massif le coefficient d'anisotropie est peu modifié $(K \# 5)$ alors que les processus de collisions électroniques changent radicalement sous l'influence des impuretés non intentionnelles. Ainsi les collisions de type coulombien l'emportent sur les collisions avec les phonons non seulement à basse température, mais aussi à la température ambiante lorsque la distance par rapport au substrat devient inférieure à $0,3 \mu \mathrm{m}$. Notons aussi qu'une partie des électrons se trouvent localisés dans des puits de potentiel de profondeur voisine de $25 \mathrm{meV}$.

Enfin une autre caractéristique du SOS est de présenter de fortes contraintes d'interface film-substrat liées au processus de croissance par hétéro-épitaxie à haute température. Ces contraintes, révélées par spectroscopie Raman et par magnétotransport sont responsables dans une large mesure de la plus faible mobilité dans les couches SOS comparativement au Si massif. Le modèle de conduction à 4 ellipsoïdes permet de résumer, sur le plan théorique, cette modification.

Toutefois, si les propriétés électriques sont dans l'ensemble bien corrélées aux propriétés structurales des couches SOS, il n'en reste pas moins que certains points demeurent encore mal perçus. Tel est le cas, par exemple, de l'origine précise de la couche de transition à l'interface $\mathrm{Si}-\mathrm{Al}_{2} \mathrm{O}_{3}$ et de son rôle universel vis-à-vis des propriétés électriques. Certes, si son caractère dégénéré à basse température ne fait aucun doute, en revanche sa nature et son mécanisme de formation sont controversés. Certains auteurs l'attribuent à l'existence de fluctuations de potentiel d'amplitude croissante vers l'interface Si-substrat. D'autres auteurs suggèrent qu'il s'agit plutôt d'une couche d'accumulation associée à la présence de charges fixes dans le saphir ou d'une zone d'inversion faible. Il est vraisemblable qu'une compréhension plus approfondie de cette couche de transition passe par la connaissance du degré d'amorphisation et plus généralement de la densité d'états des bandes dans cette région. Sur le plan expérimental cela suggère l'opportunité d'étendre les études des propriétés de transport à une gamme de température plus basse afin de faire la part entre les modèles théoriques cités.

Un autre point délicat est relatif aux états de l'interface $\mathrm{Si}-\mathrm{Al}_{2} \mathrm{O}_{3}$ dont la densité totale est bien connue (\# $10^{12} \mathrm{~cm}^{-2}$ ) mais le profil énergétique dans la bande interdite présente une dispersion. D'après certains il existe près du milieu du gap un pic, attribué à un niveau profond dans la couche de transition, alors que d'autres trouvent une densité d'états plus importante près des bords de bande, comme pour l'interface $\mathrm{Si}-\mathrm{SiO}_{2}$.

Enfin, les valeurs extrêmement faibles des mobilités au voisinage du substrat (allant jusqu'à $10 \mathrm{~cm}^{2} / \mathrm{Vs}$ ) n'ont été que qualitativement justifiées par le caractère désordonné de l'interface.

Il est bon de souligner que les mobilités tirées des caractéristiques des transistors MOS sont entachées d'une certaine imprécision dans la mesure où les seuls modèles de TMOS existant ne prennent pas en compte l'influence du désordre et, plus généralement, les phénomènes de localisation aux interfaces. Une étude plus complète des propriétés de transport à l'interface $\mathrm{Si}-\mathrm{Al}_{2} \mathrm{O}_{3}$ par effet de champ se doit d'associer la mesure de l'effet Hall dans la couche d'inversion à l'interprétation des caractéristiques des transistors MOS face arrière par un modèle physique réaliste.

\subsection{EFFiCACITÉ ET OPTIMISATION DES MÉTHODES DE} CARACTÉRISATION DU SOS. - En raison de la complexité des dispositifs CMOS sur SOS (faibles dimensions, grande densité d'intégration) il est essentiel, d'abord, de démarrer le processus d'intégration à partir de couches épitaxiées de qualité convenable et, ensuite, de suivre les propriétés du matériau durant les différents stades technologiques. Il se pose la question des critères physiques définissant une qualité minimale du matériau et celle du choix des méthodes optimales de caractérisation. Ces méthodes de test se doivent sûres et suffisamment simples pour pouvoir être appliquées au cours du flux technologique. Il est clair par exemple que pour évaluer la qualité de l'hétéroépitaxie, les techniques fondées sur le fonctionnement des dispositifs auront une efficacité limitée en raison du retard provoqué par leur fabrication et ne peuvent être exploitées que ponctuellement ; au contraire elles sont essentielles dans l'étude des perturbations apportées au matériau par les différentes étapes de l'intégration (oxydation, métallisation, recuit...). Néanmoins, la mise au point de méthodes fiables de test nécessite de multiples vérifications s'appuyant sur la confrontation de différentes mesures physico-chimiques ou électriques, dont certaines de grande complexité.

Le tableau III donne une vue synthétique des principaux paramètres physiques et des méthodes jugées comme optimales pour leur caractérisation. Les techniques pouvant apporter des informations complémentaires sont également mentionnées. Nous nous sommes aussi intéressés à l'évaluation de la précision et à la présentation des conditions particulières de mesure.

Les techniques spectroscopiques jouent un rôle considérable :

- elles permettent d'établir des relations entre les paramètres gouvernant la préparation du matériau (température de l'hétéroépitaxie, vitesse, orientation, etc...) et la qualité cristalline des couches : identification des défauts, détermination de leur orientation et de leur densité, analyse de la structure de bande et des contraintes élastiques, appréciation de l'état des surfaces et des interfaces. 
Tableau III. - Techniques de caractérisation des principales propriétés des films SOS. Précision : $\left.{ }^{* * *}\right)$ très bonne (quelques $\%$ d'erreur), ( ${ }^{* *}$ ) bonne (quelques $10 \%$ d'erreur malgré conditions expérimentales difficiles et/ou théorie complexe), $\left({ }^{*}\right)$ résultat qualitatif.

[Characterization techniques for the determination of the main properties of SOS films. Accuracy : $\left({ }^{* * *}\right)$ very good (some $\%$ of error), $\left({ }^{* *}\right)$ good (some $10 \%$ of error although experimental difficulties and/or complicated theories), $\left({ }^{*}\right)$ qualitative result].

\begin{tabular}{|c|c|c|c|c|c|}
\hline \multicolumn{2}{|c|}{$\begin{array}{l}\text { PROPRIETE } \\
\text { FONDAMENTALE }\end{array}$} & $\begin{array}{l}\text { EXPERIENCE } \\
\text { OPTIMALE }\end{array}$ & $\begin{array}{l}\text { EXPERIENEE } \\
\text { COMPLEMENTAIRE }\end{array}$ & PRECISION & $\begin{array}{c}\text { CONDITIONS } \\
\text { PARTICULIERES }\end{array}$ \\
\hline \multirow{5}{*}{ 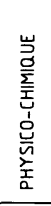 } & $\begin{array}{c}\text { NuCLEATION } \\
\text { couche de transition }\end{array}$ & $\begin{array}{l}\text { MICROSCOPIE ELEC- } \\
\text { TRONIOUE MEB MET }\end{array}$ & RAYONS $x$ & une mona ouche & diff- orientations \\
\hline & $\begin{array}{l}\text { CRISTALLINTE - } \\
\text { HOMOGENEITE }\end{array}$ & REFLECTIVITE UV & \begin{tabular}{|l|} 
TOPOGGAPAHE \\
RAYON \\
PHOTOVOTAGE
\end{tabular} & $* * *$ & $\begin{array}{l}\text { uv entre } 2-5.5 \text { eV } \\
\text { RX "rocking curve" }\end{array}$ \\
\hline & \begin{tabular}{|l|} 
TAUX et PROFLLS \\
IIMPURETES ef DEFAUTS
\end{tabular} & SIMS & AUGER & ** & - \\
\hline & \begin{tabular}{|c|} 
STRUCTURE de \\
BANDE- CONTRAINTES \\
\end{tabular} & RAMAN & $\begin{array}{l}\text { TRANSPORT } \\
\text { PHOTOVOL TAGE }\end{array}$ & ** & To. B varizbles \\
\hline & \begin{tabular}{|l} 
RUGOSITE ou \\
ETAT de SURFACE
\end{tabular} & $\begin{array}{l}\text { RAYONS } x \\
\text { REFL RASANTE }\end{array}$ & REFLECTIVITE UV & ** & uv $\quad \lambda=4,000 \dot{A}$ \\
\hline \multirow{7}{*}{ 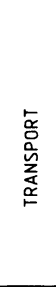 } & RESISTIVITE & CONDUCTION & PнOTO- $\sigma$ & $* * *$ & $\begin{array}{l}\text { T variable } \\
\text { cartographie }\end{array}$ \\
\hline & MOBLLITE & HALL & 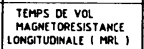 & $* * *$ & $\begin{array}{l}\text { haut } 8.1^{\circ} \\
\text { MRL }(110)\end{array}$ \\
\hline & $\begin{array}{l}\text { CONEENTRATION } \\
\text { PORTEURS }\end{array}$ & HALL & PTE & ** & $T^{\circ}$ \\
\hline & $\begin{array}{l}\text { MODE DE } \\
\text { COLLISIONS }\end{array}$ & HALL & \begin{tabular}{|l|} 
MAGNE TORESISTANCE \\
TRANSVERSALE (MRT)
\end{tabular} & * & $T \cdot$, naut B \\
\hline & NIVEAU DE FERMI & $\begin{array}{l}\text { POUVOIR TH-EL } \\
\text { (PTE) } \\
\end{array}$ & HALL & ** & $T^{\circ}$ \\
\hline & $\begin{array}{r}\text { INHOMOGENEITE } \\
\text { GLOBALE }\end{array}$ & PTE & HALL, MR & * & $T^{\circ}$, hout $B$ \\
\hline & $\begin{array}{l}\text { PROFILS } \\
\text { DINHOMOGENETE } \\
\end{array}$ & \begin{tabular}{|l} 
HALLL MOS \\
MR. CONOUCTIVITE \\
\end{tabular} & CAPACITE MOS & ** & \begin{tabular}{|l|}
$\begin{array}{l}\text { Tre et fréquence } \\
\text { vartables haut B }\end{array}$ \\
\end{tabular} \\
\hline \multirow{2}{*}{ 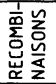 } & $\begin{array}{c}\text { OUREE OE VIE } \\
\text { VOLUMIOUE }\end{array}$ & 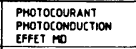 & \begin{tabular}{|l|} 
EFFET PHOTOMAGNEE \\
TOELECTRIQUE (PME)
\end{tabular} & ** & $\begin{array}{c}\text { Laser, } \\
\text { haut } \\
\text { hou }\end{array}$ \\
\hline & \begin{tabular}{|c|} 
ViTESSE DE RECOMB \\
EN SURFACE
\end{tabular} & MAGNETODIOOE (MD) & $\begin{array}{l}\text { DIODES CONTROLEES } \\
\text { PAR } 1 \text { O OU } 2 \text { GRILLES }\end{array}$ & ** & hout $B$ \\
\hline \multirow{4}{*}{ 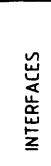 } & \begin{tabular}{|l} 
DENSITE DETATS \\
DENSITE OE CHARGES \\
\end{tabular} & $\begin{array}{c}\text { CAPACIIT MOS } \\
\text { CIV) }\end{array}$ & $\begin{array}{l}\text { TMOS } \\
\text { BRUIT }\end{array}$ & ** & 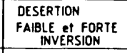 \\
\hline & \begin{tabular}{|l} 
MOBLIITE DANS \\
CANAL INVERSION \\
\end{tabular} & TMOS & 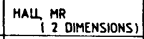 & *** & T et $B$ variables \\
\hline & $\begin{array}{l}\text { MASSE EFF DE } \\
\text { SURFACE- ANISOTROPIE }\end{array}$ & \begin{tabular}{|l} 
SHUBNIKOV \\
DE HAAS $/$ TMOS
\end{tabular} & RAMAN & ** & haut 8 \\
\hline & $\begin{array}{l}\text { MOBLITE INTERFACE } \\
\text { S1-A1203 }\end{array}$ & \begin{tabular}{|l} 
MMOS \\
c(V)
\end{tabular} & \begin{tabular}{|l|} 
PTE \\
PHOTOCONDUCTIVITE \\
\end{tabular} & * & $\begin{array}{l}\text { Amincissement du } \\
\text { substrat }\end{array}$ \\
\hline
\end{tabular}

- la précision des mesures est généralement bonne malgré certaines difficultés pratiques (préparation très minutieuse des échantillons, effets parasites dus à la rugosité des surfaces, etc...).

\section{Néanmoins :}

- la grande complexité de ces techniques les rend inexploitables de manière systématique dans une filière technologique,

- les résultats déduits ne sont que très indirectement corrélables aux performances des dispositifs à réaliser.

Dans un but de simplification il a été récemment proposé $[100,101]$ d'utiliser la mesure de la variation du coefficient de réflexion des rayons UV (à $\lambda=2800 \AA$ et $\lambda=4000 \AA$ ) pour la détermination du pourcentage de défauts dans le SOS et de l'irrégularité de la surface. La fiabilité de cette méthode, qui permet en plus une topographie bidimensionnelle des couches, a été vérifiée par microscopie électronique en transmission et par spectroscopie à rayons $\mathrm{X}$ [100].

Les paramètres macroscopiques tels la mobilité, la concentration, l'anisotropie, sont déduits à l'aide de méthodes de transport électrique. Malgré l'éventail des conditions de mesure (température, champ magné- tique, photo-excitation, ...) les erreurs expérimentales restent faibles, les principales limitations étant d'ordre fondamental :

- détermination de propriétés inhomogènes par mesures effectuées sur toute la couche,

- équivalence injustifiée entre mobilités de Hall et de dérive, entre mobilités de volume et de surface, entre densités d'impuretés ionisées et de porteurs mobiles,

- utilisation de modèles théoriques inadaptés,

- gammes insuffisantes de mesure (température, fréquence, ...).

Les données qui apparaissent indispensables pour une caractérisation globale, même très simplifiée du SOS, sont bien sûr la conductivité et l'effet Hall mais aussi le pouvoir thermoélectrique.

L'efficacité des structures à dépletion (MOS-Hall, capacité, ...) dans la détermination des profils verticaux des paramètres de transport est reconnue mais des limitations subsistent toutefois :

- nécessité d'un faible dopage favorisant une déplétion profonde,

- existence de la longueur de Debye et d'autres sources d'erreurs qui peuvent être minimisées sur le plan pratique par la détermination de la tension de bande plate pour chaque température, par le choix de la fréquence de mesure et d'un schéma électrique équivalent réaliste.

C'est l'ensemble de ces précautions qui assurent une faible marge d'erreur (5 à 10\%) aux résultats déduits (profils de mobilités, d'anisotropie, de concentration, ...) et, conduisent en contrepartie à une complexité expérimentale telle que l'automatisation de la mesure devient souhaitable. Une méthode duale pour la mesure des profils consiste à réduire progressivement l'épaisseur des couches par attaque chimique, évitant ainsi les problèmes liés au processus de déplétion; l'inconvénient essentiel vient de l'appréciation des propriétés de volume à partir de la mesure (différentielle) des contributions de couches situées en surface et contaminées, en plus, par l'attaque.

Les paramètres déduits du fonctionnement des dispositifs intégrés sont les plus suggestifs quant à la qualité du circuit final; c'est aussi par eux que l'on mesure en pratique l'efficacité d'une optimisation technologiques. Comme ces paramètres peuvent être affectés d'incertitudes expérimentales, dues par exemple à l'inhomogénéité horizontale des plaques, il est préférable d'utiliser des histogrammes topographiques de résultats [100]. Mais les incertitudes se manifestent plutôt durant la phase de modélisation : bien des aspects théoriques ne sont pas convenablement maîtrisés (bruit $1 / f$, piégeage d'électrons chauds dans l'oxyde, mécanismes des courants de fuite à l'interface $\left.\mathrm{Si}-\mathrm{Al}_{2} \mathrm{O}_{3}, \ldots\right)$ et de nombreux modèles parallèles coexistent, conduisant à des résultats différents. L'utilisation de modèles repose aussi sur la présomption que les paramètres auxiliaires sont connus avec pré- 
cision. A titre d'exemple, dans le cas de l'exploitation d'un modèle simplifié de l'effet magnétodiode, il a été montré qu'une faible incertitude (10 à $50 \%$ ) sur les mobilités et/ou sur le dopage engendre des erreurs très fortes (100 à $1000 \%)$ sur l'estimation de la durée de vie et de la vitesse de recombinaison à l'interface $\mathrm{Si}-\mathrm{Al}_{2} \mathrm{O}_{3}$ [91]; pour éviter cet inconvénient il a fallu faire appel à un modèle plus rigoureux et surtout à des données expérimentales supplémentaires $[52,96]$.

Les résultats obtenus dans les tableaux II et III permettent d'établir un organigramme du processus de caractérisation (Fig. 4.1). Ce schéma montre les possibilités existant pour compléter et optimiser les études sur SOS, ainsi que les éléments à considérer pour amorcer une étude cohérente des couches de $\mathrm{Si}$ sur $\mathrm{SiO}_{2}$.

4.3 ConClusion. - Nous avons présenté dans ce travail les propriétés des couches SOS fabriquées en France, provenant, en grande partie, des recherches menées par de nombreuses équipes dans le cadre d'une action concertée (GCIS). Les résultats obtenus par des techniques très différentes montrent un bon degré de corrélation. Les quelques questions restant ouvertes concernent tout particulièrement la qualité de l'interface $\mathrm{Si}_{-} \mathrm{Al}_{2} \mathrm{O}_{3}$ [102] et les moyens proposés pour diminuer le nombre de défauts : [103],

- écrantage de l'interface par surdopage profond

- réduction des contraintes à $300 \mathrm{~K}$ par création artificielle (laser) de contrainte «négative» à la température du dépôt [104],

- hydrogénation des couches [105].

En ce qui concerne l'avenir du SOS il semble que le pessimisme qui régnait les années précédentes était exagéré [106], car de nouveaux circuits continuent d'apparaître [5, 106]. La résistance du SOS aux irradiations le rend indispensable pour de nombreuses applications. Par ailleurs, la plus faible mobilité dans

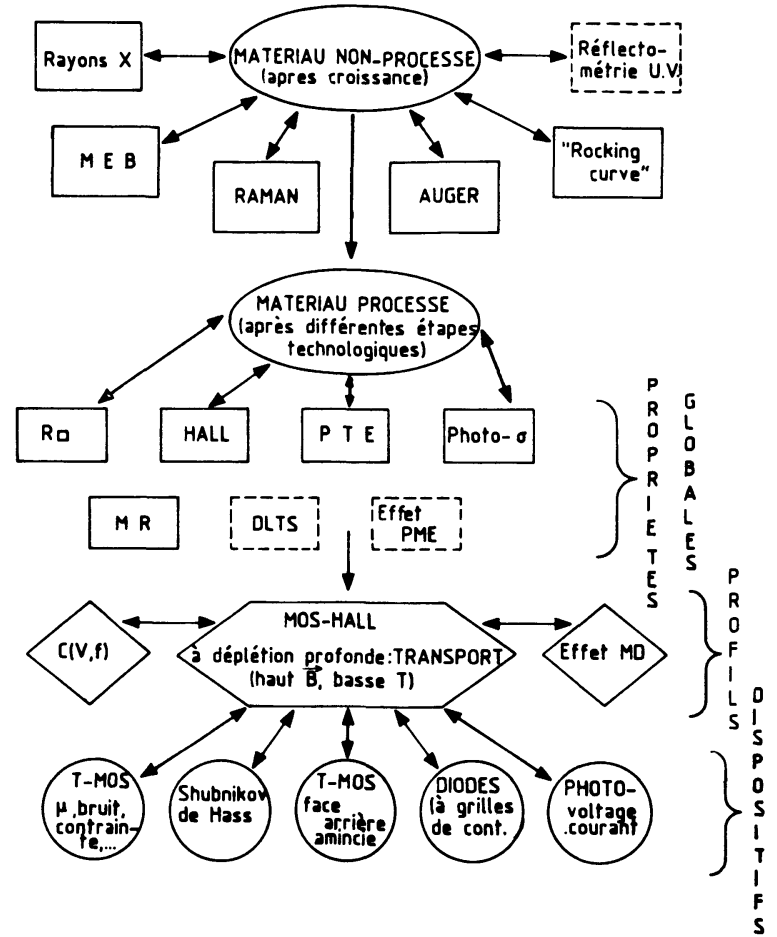

Fig. 4.1. - Organigramme du processus préconisé pour la caractérisation des technologies Silicium Sur Isolant; en cadre plein mesures déjà exploitées pour le SOS.

[Organization of a suitable characterization process for Silicon On Insulator; full lines show the techniques already exploited for SOS.]

le SOS n'est plus un inconvénient majeur lorsqu'on pense aux dispositifs sub-microniques à échauffement de porteurs. Enfin, les couches de $\mathrm{Si}$ sur $\mathrm{SiO}_{2}$, dont les techniques de fabrication ne sont pas encore certaines, ne paraissent pas vouées à concurrencer le SOS [107]; elles seront vraisemblablement destinées à des applications différentes concernant en particulier le développement des circuits à 3 dimensions.

Bibliographie

[1] Borel, J., Onde Electrique 58 (1978) 812.

[2] Micheletti, F. B., IEEE Trans. Electron. Devices ED 25 (1978) 857.

[3] Maurits, J. E., IEEE Trans. Electron. Devices ED 25 (1978) 859.

[4] Cullen, G. W., Wang, C. C., Heteroepitaxial Semiconductors for Electronic Devices (Springer Verlag. New York) (1978)

[5] 1982 IEEE SOS/SOI Technology Workshop. Provincetown, USA (1982).

[6] Fraimbault, J. L., Thèse d'Etat, USM Grenoble (1967).

[7] Trilhe, J., Peccoud, L., Verdone, M., Rapport LETI-DRME 72.34-415.00.480.7501 (1973).

[8] Cristoloveanu, S., Ghibaudo, G., Kamarinos, G., 1982 IEEE SOS/SOI Technology Workshop, Provincetown, USA (1982).
[9] Cristoloveanu, S., Séminaire Franco-Britannique « Le Matériau Silicium », CNET Grenoble (1982).

[10] Trilhe, J., Montier, M., Sollier, A., Rapport LETI/MEA no 1087 (1975).

[11] Trilhe, J., Le Gal, H., Rolland, G., Rapport LETI/ MEA no 1093 (1975).

[12] Le Gal, H., TASSON, M., Rapport LETI/CRM no 77 1261 (1977).

[13] Gonchond, J. P., Thèse USM Grenoble (1978).

[14] Croce, P., Acta Electron. 24 (1981/1982) 247.

[15] Croce, P., Nevot, L., Rapport GCIS (1979).

[16] Carfagnini, M. L., Thèse $3 \mathrm{e}$ cycle, INP Grenoble (1981).

[17] Trilhe, J., Thèse d'Etat, USM Grenoble (1975).

[18] Schwebel, C., Vapaille, A., Bouchier, D., GautheRIN, G., Meyer, F., Proc. 8th Int. Vacuum Congress, Cannes (France), vol. 1, 1980, 125. 
[19] Hamar-Thibault, S., Revue Phys. Appl. 16 (1981) 317.

[20] Englert, T., Abstreiter, G., Pontcharra, J., Sol. State Electron. 23 (1979) 31.

[21] Gonchond, J. P., Rolland, G., Trilhe, J., Rapport LETI/MEP no 1214 (1977).

[22] Trilhe, J., Blanchard, B., Borel, J., Rapport LETI/MEA no 1231 (1977).

[23] TRILHE, J., Rapport LETI/MEA no 1362 (1979).

[24] Trilhe, J., Blanchard, B., Borel, J., Proc. 3rd Int. Conf. on Solid Surfaces, Vienna (Austria), 1977, 541.

[25] Nishi, Y., Hara, Y., Japon J. Appl. Phys. Supp. 17 (1978) 27.

[26] Schlotterer, H., Solid State Electron. 11 (1968) 947.

[27] Hughes, A. J., Thorsen, A.C., J. Appl. Phys. 44 (1973) 2304.

[28] HyneceK, J., J. Appl. Phys. 45 (1974) 2631.

[29] Hughes, A. J., J. Appl. Phys. 46 (1975) 2849.

[30] Ohmura, Y., J. Phys. Soc. Japan 39 (1975) 549.

[31] Ohmura, Y., J. Phys. Soc. Japan 47 (1979) 145.

[32] LEE, J. H., Thèse Docteur-Ingénieur, INP Grenoble (1981).

[33] Shibuya, M., Phys. Rev. 95 (1954) 1385.

[34] Vapaille, A., Physique des Dispositifs à Semiconducteurs (Masson, Paris) 1970.

[35] Herring, C., Vogt, E., Phys. Rev. 101 (1955) 944.

[36] Lee, J. H., Cristoloveanu, S., Chovet, A., Solid State Electron. 25 (1982) 947.

[37] Cristoloveanu, S., Thèse d'Etat, INP Grenoble (1981).

[38] Cristoloveanu, S., Lee, J. H., Chovet, A., Revue Phys. Appl. 15 (1980) 725.

[39] Robert, J. L., Dusseau, J. M., Girard, P., Sicart, J., J. Appl. Phys. 54 (1983) 1903.

[40] Ghibaudo, G., Kamarinos, G., Revue Phys. Appl. 17 (1982) 133.

[41] Roux, M., Bielle Daspet, D., Revue Phys. Appl. 16 (1981) 495

[42] GRIS, Y., Bello, Y., Rapport LETI/MEA no 1273 (1978).

[43] Ghibaudo, G., Hsia, C. Y., Kamarinos, G., a) ESSDERC 80, York (U.K.), 1980; b) IEEE SOS Workshop, Cupertino (USA), 1980.

[44] Ghibaudo, G., Hsia, C. Y., Kamarinos, G., Proc. 8th Int. Vacuum Congress, Cannes (France), 1980 , vol. $1,459$.

[45] Ghibaudo, G., Thèse Docteur-Ingénieur, INP Grenoble (1981).

[46] Ipri, A. C., J. Appl. Phys. 43 (1972) 2770.

[47] Morin, F. J., Maita, J. P., Phys. Rev. 96 (1955) 28.

[48] Dumin, D. J., Ross, E. C., J. Appl. Phys. 41 (1970) 3139.

[49] Ipri, A. C., Zemel, J. N., J. Appl. Phys. 44 (1973) 744.

[50] Heiman, F. P., Appl. Phys. Lett. 8 (1966) 31.

[51] Elliot, A. B., ANderson, J. C., Solid State Electron. 15 (1972) 531.

[52] Cristoloveanu, S., Chovet, A., Kamarinos, G., Solid State Electron. 21 (1978) 1563.

[53] Ghibaudo, G., Tsamakis, D., Papatriantafillou, C., Kamarinos, G., Rokofillou, E., J. Phys. $C$. 16 (1983) 4479.

[54] Yasuda, Y., Ohmura, Y., Japan J. Appl. Phys. 8 (1969) 1098.

[55] Hsu, S. T., Scott, J. H., RCA Rev. 36 (1975) 240.

[56] SzE, S. M., Physics of Semiconductor Devices (Wiley, New York) 1982.
[57] Lee, J. H., Cristoloveanu, S., Proc. 8th Int. Vacuum Congress, Cannes (France), 1980, 550.

[58] Lee, J. H., Cristoloveanu, S., ESSDERC \& SSSDT, Toulouse (France) 1981.

[59] Lee, J. H., Cristoloveanu, S., Chovet, A., Thin Solid Films 89 (1982) 13.

[60] LEE, J. H., Electron. Lett. 17 (1981) 566.

[61] Gentil, P., Revue Phys. Appl. 13 (1978) 609.

[62] Hsu, S. T., ScOTt, J. H., HAM, W. E., IEDM, Washington (USA), 1973.

[63] Le Goascoz, V., Borel, J., Suat, J., ESSDERC, Nottingham (U.K.), 1974.

[64] Rigaud, D., Touboul, A., Sodini, D., Lopez, J. C., LeCoy, G., Revue Phys. Appl. 15 (1980) 937.

[65] Gris, Y., Montier, M., Rapport LETI/MEA no 1306 (1978).

[66] Bernard, J., Garcia, M., Suat, J. P., Rapport LETI/MEA no 888 (1972).

[67] MonRoy, A., Rapport LETI/MEA no 1321 (1978).

[68] GaRCIA, M., Thèse Docteur-Ingénieur, USM Grenoble (1974).

[69] Kawaji, S., Hatanara, K., Nakamura, K., Onga, S., J. Phys. Soc. Japan 41 (1976) 1073.

[70] Englert, T., Landwher, G., Pontcharra, J., DorDA, G., Surface Sci. 98 (1980) 427.

[71] Sun, S. C., Plummer, J. D., IEEE Trans. Electron. Devices ED 27 (1980) 1497.

[72] Gentil, P., Chausse, S., Solid State Electron. 20 (1977) 935.

[73] ChoujaA, A., Thèse Docteur-Ingénieur, INP Grenoble (1977).

[74] Touboul, A., Peloux, G., Lecoy, G., ChoujaA, A., Gentil, P., Reviue Phys. Appl. 13 (1978) 227.

[75] Goodman, A. M., IEEE Trans. Electron. Devices ED 21 (1974) 753 et ED 22 (1975) 63.

[76] Nicollian, E. H., Goetzberger, A., Bell System J. 46 (1967) 1055.

[77] Castagne, R., Thèse d'Etat, Paris (1970).

[78] Rossel, P., Thèse d'Etat, Toulouse (1973).

[79] Dube, C. Krusius, P., Frey, J., SOS Workshop, Snowmass (USA), 1980.

[80] GeNTIL, P., Electron. Lett. 16(1980) 617.

[81] MoreaU, F., Rapport DEA, ENSER Grenoble (1980).

[82] BRINI, J., Gentil, P., Rapport EFCIS-ENSER Grenoble (1983)

[83] Dumin, D. J., Solid State Electron. 13 (1970) 415.

[84] Lagowski, J., Jastrzebski, L., Cullen, G. W., J. Electrochem. Soc. 128 (1981) 2665.

[85] Dumin, D. J., Silver, R. S., Solid State Electron. 11 (1968) 353.

[86] Norris, C. B., Appl. Phys. Lett. 20 (1972) 187.

[87] Aвrahams, M. S., Buiocchi, C. B., Appl. Phys. Lett. 27 (1975) 325.

[88] McGreivy, D. J., Viswanathan, R. C., Appl. Phys. Lett. 25 (1974) 505.

[89] Schroder, D. K., Rai-Chandouri, R., Appl. Phys. Lett. 22 (1973) 455.

[90] SENN, P., Thèse Docteur-Ingénieur, INP Grenoble (1975).

[91] Cristoloveanu, S., Kamarinos, G., Viktorovitch, P., Borel, J., Staderini, R., Proc. 7th Int. Vacuum Congr. \& 3rd Int. Conf. on Solid Surfaces, Vienna (Austria), 1977, 1943.

[92] Lilienkamp, P., Pfleiderer, H., Phys. Status Solidi (a) 43 (1977) 479 . 
[93] Cristoloveanu, S., Phys. Status Solidi (a) 64 (1981) 683 et 65 (1981) 281.

[94] Mohaghegh, A., Cristoloveanu, S., Pontcharra, J., IEEE Trans. Electron. Devices ED 28 (1981) 237.

[95] Kranzer, D., Appl. Phys. Lett. 25 (1974) 103.

[96] Cristoloveanu, S., Proc. Int. Conf. on Appl. of High Magnetic Fields in Semicond. Phys., Grenoble (France), 1982, 427.

[97] Dufour, M., Cristoloveanu, S., 2nd Annual Gen. Cond. Matter Divis. Conf. of the EPS, Manchester (Angleterre), 1982.

[98] Dufour, M., Cristoloveanu, S., Appl. Phys. A 29 (1982) 87.

[99] Jacoboni, C., Canali, C., Ottaviani, G., Alberigi Quaranta, A., Solid State Electron. 20 (1977) 77.

[100] Cullen, G. W., Abrahams, M. S., Corboy, J. F., Duffy, M. T., HAM, W. E., JASTRZebski, L.,
Smith, R. T., Blumenfeld, M., Harbeke, G., LAGowski, J., J. Crystal Growth 56 (1982) 281.

[101] JASTRzebSKi, L., Duffy, M. T., Corboy, J. F., Cullen, G. W., LagowsKi, J., J. Crystal. Growth 58 (1982) 37.

[102] Ponce, F. A., Appl. Phys. Lett. 41 (1982) 371.

[103] Yamamoto, Y., SugiYama, T., Hara A., Inada, T., J. Appl. Phys. 53 (1982) 793.

[104] Sai-Halasz, G. A., Fang, F. F., Sedgwick, T. O., Segmuller, A., Appl. Phys. Lett. 36 (1980) 419.

[105] Jastrzebski, L., Lagowski, J., Cullen, G. W., Pankove, J. I., Appl. Phys. Lett. 40 (1982) 713.

[106] Pancholy, R. K., 1982 IEEE SOS/SOI Technology Workshop, Provincetown (USA), 1982.

[107] JASTRZEBSKI, L., 1982 IEEE SOS/SOI Technology Workshop, Provincetown (USA), 1982. 